\title{
A Dynamic Phase-field Model for Structural Transformations and Twinning: Regularized Interfaces with Transparent Prescription of Complex Kinetics and Nucleation. Part I: Formulation and One-Dimensional Characterization.
}

\author{
Vaibhav Agrawal* and Kaushik Dayal ${ }^{\dagger}$ \\ Carnegie Mellon University
}

March 27, 2015

\begin{abstract}
The motion of microstructural interfaces is important in modeling twinning and structural phase transformations. Continuum models fall into two classes: sharp-interface models, where interfaces are singular surfaces; and regularized-interface models, such as phase-field models, where interfaces are smeared out. The former are challenging for numerical solutions because the interfaces need to be explicitly tracked, but have the advantage that the kinetics of existing interfaces and the nucleation of new interfaces can be transparently and precisely prescribed. In contrast, phase-field models do not require explicit tracking of interfaces, thereby enabling relatively simple numerical calculations, but the specification of kinetics and nucleation is both restrictive and extremely opaque. This prevents straightforward calibration of phase-field models to experiment and/or molecular simulations, and breaks the multiscale hierarchy of passing information from atomic to continuum. Consequently, phase-field models cannot be confidently used in dynamic settings.

This shortcoming of existing phase-field models motivates our work. We present the formulation of a phase-field model - i.e., a model with regularized interfaces that do not require explicit numerical tracking - that allows for easy and transparent prescription of complex interface kinetics and nucleation. The key ingredients are a re-parametrization of the energy density to clearly separate nucleation from kinetics; and an evolution law that comes from a conservation statement for interfaces. This enables clear prescription of nucleation - through the source term of the conservation law - and kinetics - through a distinct interfacial velocity field. A formal limit of the kinetic driving force recovers the classical continuum sharp-interface driving force, providing confidence in both the re-parametrized energy and the evolution statement. We present some 1D calculations characterizing the formulation; in a companion paper, we present more extensive 2D calculations.
\end{abstract}

Keywords: Phase-field modeling, Twinning, Structural phase transformation, Nucleation of Interfaces, Kinetics of Interfaces

*vaibhavalandrew. cmu.edu

$\dagger$ kaushikecmu.edu 


\section{Introduction}

Twinning and structural phase transformations are important in areas as diverse as superelasticity and shape-memory in functional materials [Bha03], forming of structural metals [BCR10], nanostructured metals with exceptional properties such as high strength and high ductility [HB14, KAF09, WTAF09], and the dynamic response of metals under extreme conditions [CSWRS09]. The typical microstructure in these settings consists of homogeneously deformed regions separated by interfaces across which the deformation varies extremely rapidly. Many important aspects of these phenomena are governed by the nucleation, motion, and response of the interfaces.

In the continuum setting, twinning and structural transformations are modeled using nonconvex strain energy density functions $W(\epsilon)$, an approach introduced in the seminal paper of Ericksen [Eri75] in 1D. The nonconvexity allows for the coexistence of different phases or twins for a given stress value $\sigma=\frac{d W}{d \epsilon}$. The different phases are separated by interfaces across which the strain is discontinuous. Since the standard continuum theory contains no lengthscale, these interfaces are "sharp", i.e. singularly localized. Ericksen observed that the continuum balance of linear momentum is insufficient to identify a unique spatial location of the interfaces, even assuming the existence of a single interface. In the static setting without inertia, he used energy minimization as a selection criterion to obtain a unique solution.

Abeyaratne and Knowles [AK90, AK91b] examined nonconvex models in the dynamic setting with inertia. Again, balance of linear momentum does not provide a unique solution even in the simplest case of a single interface in 1D. Further, energy minimization is not applicable in dynamic problems and cannot be used to resolve this. Invoking thermodynamics, viz. positive dissipation, provides some weak restrictions on the motion of the interface, but still leaves a massively nonunique problem with essentially a 1-parameter family of solutions that is parametrized by the location of the interface. [AK90, AK91b], and related work in [Tru82], find that imposing additional closure relations makes the problem unique. Namely, the closure relations are (i) the kinetic relation that relates the velocity of the interface to the thermodynamic work conjugate driving force, and (ii) the nucleation criterion that provides for the formation of new interfaces. Physically, the closure relations can be thought of as a macroscopic remnant of the lattice-level atomic motion from one energy well to another that is lost in the continuum theory. However, a systematic derivation from a microscopic theory as well as experimental confirmation remain a topic of active research.

The closure relations - nucleation criterion and kinetic relation - have the advantage of clear and direct physical interpretations. In particular, they fit naturally into a multiscale modeling framework by allowing for precisely-defined constitutive input on the behavior of interfaces from either experiment or modeling (e.g., molecular dynamics). However, numerical computations with this approach are extremely challenging, because the sharp interfaces require complex and expensive tracking algorithms in a numerical discretization. This is an unfeasible challenge when one expects numerous interfaces that are evolving, interacting, and nucleating. Therefore, this sharp-interface approach has not been widely applied to larger problems.

In contrast to this, there is a large body of work on methods that regularize or smooth the interface by adding strain gradients, viscous dissipation, and similar effects to the stress response, e.g. [AK91a, Ros95, Tur97, Tru93, FM06]. In 1D, the stress in these models is typically given by:

$$
\sigma=\frac{d W}{d \epsilon}+\nu \frac{\partial \epsilon}{\partial t}-\kappa \frac{\partial^{2} \epsilon}{\partial x^{2}}
$$

In these regularized-interface approaches, the evolution of interfaces is obtained simply by solving mo- 
mentum balance $\operatorname{div} \sigma=\rho \ddot{u}$. The solutions are typically unique but depend strongly on the regularization parameters, viz. the viscosity $\nu$ and the capillarity $\kappa$. While the nonconvexity in $W$ favors the formation of interfaces, the gradient regularization $\kappa \frac{\partial^{2} \epsilon}{\partial x^{2}}$ penalizes the sharpness of interfaces and thereby prevents them from being singular. Because interfaces are not singular and hence do not need to be explicitly tracked, these approaches are relatively easy to apply to large problems. Further, nucleation of new interfaces and topology transitions occur naturally without additional computational effort or constitutive input.

In the closely-related phase-field approaches, the situation is similar. The phase are distinguished by a scalar field $\phi$, and the energy is nonconvex in $\phi$ with a coupling to elasticity. A typical phase-field energy density [Che02, SL07, ZB05, AA12, YD10, SY08, LLSL10] has the form

$$
w(\phi)+\frac{1}{2}\left(\boldsymbol{\epsilon}-\boldsymbol{\epsilon}_{0}(\phi)\right): \boldsymbol{C}:\left(\boldsymbol{\epsilon}-\boldsymbol{\epsilon}_{0}(\phi)\right)+\kappa|\nabla \phi|^{2}
$$

$w(\phi)$ is a nonconvex energy and favors the formation of interfaces, while the gradient term $\kappa|\nabla \phi|^{2}$ regularizes them. These terms are coupled to linear elasticity through the elastic strain $\left(\boldsymbol{\epsilon}-\boldsymbol{\epsilon}_{0}(\phi)\right)$, that is the difference between the total strain $\epsilon \equiv \operatorname{grad} \boldsymbol{u}$ and the stress-free strain $\epsilon_{0}(\phi)$ that depends on the phase. The total energy $E$ is obtained by integrating the energy density over the body and accounting for the boundary working. The evolution is governed by a gradient descent in $\phi$, i.e. $\mu \dot{\phi}=\frac{\delta E}{\delta \phi}$, and linear momentum balance for the evolution of the displacement / strain field. Phase-field models share the key features of the strain-gradient models:

1. the evolution of interfaces is unique, and governed by $\mu, \kappa$ and other model parameters;

2. nucleation and topology transitions occur naturally without additional input, and like kinetics, are governed by $\mu, \kappa$ and other model parameters;

3. nucleation and kinetics are modeled together in a single equation; and

4. they are relatively easy to apply to large problems because interfaces are not singular.

Feature 4 of phase-field models is an important advantage of these approaches. However, Features 1, 2 , and 3 are not advantages, but instead important shortcomings of these models. While it is certainly important to obtain unique solutions, the fact that the nucleation and kinetics of interfaces are governed by a small set of parameters implies that the range of behavior that can be modeled is highly restricted. In addition, the nucleation and kinetics of interfaces are physically distinct processes from the atomic perspective, but in these models are governed through the same evolution equation.

Feature 1 greatly limits the ability to formulate a model that produces a desired kinetic response. For example, the kinetics of interfaces in strain-gradient models is analyzed by [AK91a], and they find that the range of kinetic responses that can be obtained by varying $\nu$ and $\kappa$ is extremely constrained ${ }^{1}$. For instance, an important feature that is widely observed is stick-slip behavior of interfaces, e.g. [FS11], but this cannot be readily modeled in strain-gradient or phase-field models. In general, it is difficult to prescribe a given kinetic response directly; making the parameter $\mu$ a function of various quantities may allow this, but the dependence on these quantities to obtain a desired kinetic response is opaque. Therefore, calibrating a desired kinetic response using this route can require much trial-and-error that is tedious, unsystematic, and very expensive.

\footnotetext{
${ }^{1}$ In generalized versions, e.g. [FG94, Ros95, GF97], a larger class of kinetics is possible, but the relation between the model parameters and the induced kinetics is often not transparent even for 1D.
} 
The situation in Feature 2 is similar to that in Feature 1, except that it is much more difficult! Existing phase-field models are completely opaque, even in 1D, about the precise critical condition at which nucleation occurs [DB06]. The nucleation behavior in a three-dimensional setting, with a complex energy landscape and numerous local extrema, combined with both inertial and gradient descent dynamics, is even more complex. Consequently, the inverse problem is extremely hard: namely, how do we set up the energy and evolution to obtain a desired nucleation response? That is, given some critical conditions under which nucleation takes place - perhaps from experimental observation or molecular calculations how do we tailor the various functions and parameters in the model to achieve this behavior? Modifying the energy barriers is an obvious starting point, but is difficult to do systematically; for instance, changing an energy barrier affects the nucleation behavior of both the forward and reverse transformations. In a situation with numerous possible transformations, modifying the barrier can have unintended effects on all the transformations. Another strategy is to use spatially-localized defects (or "soft spots", e.g. [ZB05]), where the energy landscape is locally modified to have shallow barriers to aid nucleation. This approach is difficult to use in situations that have not already been well-characterized by other techniques. For instance, to obtain a desired nucleation stress, how should the soft spots be spatially arranged? should we have more soft spots with higher barriers, or a few soft spots with lower barriers? what shape should they be? and so on. The current approach is typically ad-hoc, and involves trying a given configuration of soft spots, and doing full-field calculations to test if the given configuration provides the desired nucleation behavior. Other strategies to induce nucleation, such as adding external driving noise, differ in the details, but have the same basic problem that modeling a desired nucleation behavior essentially requires solving an unwieldy inverse problem posed in a very large space in an unsystematic and expensive way, when the forward problem itself is not well-understood. In addition, this inverse problem can be highly geometry- and problem-specific; calibration for a specific geometry will likely not be transferable to other geometries. This difficult situation is vastly compounded when one begins to consider the realistic case that there is not simply one type of twinning interface, but rather various different ones for orientations, each with a different propensity to nucleate and move, with the entire problem posed in 3D. Further, it is likely that critical conditions for nucleation in real systems is not simply related to the energy conjugate driving force; rather, there is likely rate-dependence, possibly dependence on hydrostatic stress even in volume-preserving twinning transformations, and so on.

Feature 3 complicates the process of calibrating a model to an observed nucleation and kinetics because the separation between these processes in the model is largely absent.

The failings of existing phase-field models make them impossible to use in a hierarchical multiscale setting. Hierarchical multiscale approaches rely on the passage of information from fundamental models to larger-scale models. In the setting of structural transformations, atomic calculations (e.g., [DWWR14, OSPM14, WBT10, BT10, BEKT12]) as well as experiments (e.g., [NRC06, EC93]) have provided important information on twin kinetics and nucleation. But almost none of this information can be used in the existing phase-field models, beyond some minor calibrations. This wastes the wealth of insights that have been gathered from atomistics and experiment, and breaks the multiscale link between the atomic level and the continuum.

The advantages and failings of the different approaches described above provide the motivation for our work. We present a regularized-interface model that has the advantage that computations are easy and efficient because we do not need to track interface evolution, nucleation, and topology transitions. However, our formulation is also designed to obtain the key advantage of the sharp interface formulation, namely that we can transparently, precisely, and readily specify complex nucleation and kinetics behavior. The technical strategy consists of 2 elements: (i) parametrization of the energy in a specific way, and 
(ii) evolution of $\phi$ through a geometric conservation law.

The first element is to re-parametrize the energy density so that it continues to reproduce the elastic response of each phase away from energy barriers, but leads to a clear separation between kinetics and nucleation. Briefly, the re-parametrized energy density ${ }^{\circ}(\boldsymbol{F}, \phi)$ is independent of $\phi$ except when $\phi$ is in a narrow range that can be considered to be the transition between the phases; an example is shown in Fig. 1 and explained in detail below. Therefore, the work-conjugate driving force for $\phi$ vanishes when $\phi$ is away from the transition range, and consequently $\phi$ cannot evolve irrespective of the value of stress and other mechanical quantities. Hence, when a region of the body is in a single phase, i.e. $\phi$ is uniform in a region, then $\phi$ cannot evolve and a new phase cannot nucleate. The only region where $\phi$ can evolve is when it is in the transition range, which occurs near an interface. Therefore, the energy allows a material region to undergo a transformation only when an interface sweeps over it, and nucleation of a new phase away from an interface is completely prohibited. We will re-introduce nucleation through the balance law in a separate term from the kinetics; the advantage of this approach is that nucleation cannot occur through the kinetic law. Thereby, our approach makes a clear distinction between kinetics and nucleation as mechanisms for the evolution of $\phi$ : the kinetic law cannot cause nucleation, and the nucleation term does not affect kinetics. This is in sharp contrast to standard phase-field models where a uniform phase may nucleate a new phase if the driving force for kinetics is sufficiently large, even if the desired critical conditions for nucleation have not been met. There, the variational derivative of the energy with respect to $\phi$ governs both the kinetics of existing interfaces as well as the nucleation of new phases. Therefore, the process of nucleation is intimately and opaquely mixed in with the prescribed kinetics in these models, making it hard to prescribe precise nucleation criteria.

The second element is to use a geometrically-motivated conservation law to govern the evolution of $\phi$. Briefly, we interpret $\nabla \phi$ as a geometric object that provides us with the linear density of interfaces. Then, for a material line element, we count the number of interfaces that are entering and leaving at each end of the element. The statement of the conservation law is that the increase in the number of interfaces threaded by the line element is equal to the net number of interfaces that are entering, plus the creation of interfaces through a source term. The motion of interfaces is described by an interface velocity field $v_{n}^{\phi}$, distinct from the material velocity field. The value of $v_{n}^{\phi}$ at each point can have a complex functional dependence on any mechanical field, e.g. stress, stress rate, nonlocal quantities, and so on, and this provides a route to transparently specify extremely complex kinetic response. Similarly, the source term in the balance law provides transparent and precise control on the nucleation of new interfaces, by activating the source only when the critical conditions for nucleation are realized. An important element is that the kinetic term is multiplied by $|\nabla \phi|$; therefore a uniform phase will not show any evolution of $\phi$ due to the kinetic term regardless of the stress level, and the only possible mechanism for the evolution of $\phi$ from a uniform state is by nucleation.

\subsection{Organization}

The paper is organized as follows.

- In Section 2, we describe the re-parametrization of the energy, the formulation of the interface balance principle, and the driving forces on interfaces obtained by enforcing positive dissipation. We also examine formally the sharp-interface limit of the dissipation in our model.

- In Section 3, we examine in 1D the behavior of steadily-moving interfaces in our model using 
a traveling-wave approach to show the relation between the prescribed kinetic response and the effective kinetics in terms of interface velocity and classical driving force.

- In Section 4, we perform 1D dynamic calculations to understand the evolution of interfaces. As in the section on traveling waves, we aim to find the effective kinetic relation induced by our model.

- In Section 5, we examine the effect of a small parameter that has been introduced in the reparametrization of the energy.

- In Section 6, we examine the competition between thermodynamics and momentum balance in setting the kinetics of an interface. We point out the difficulty our model has in dealing with certain phase interfaces whose evolution is uniquely described by momentum balance and that therefore does not require an additional kinetic relation.

- In Section 7, we review our work.

- In Appendix A, we examine briefly the connection to Noether's principle.

- In Appendix B, we examine the possibility of supersonic phase interfaces in standard phase-field models.

\subsection{Notation and Definitions}

Boldface denotes vectors and tensors. We have used Einstein convention, i.e. repeated indices imply summation over those indices, except when noted. 


\begin{tabular}{|c|c|}
\hline$\phi$ & phase field \\
\hline $\boldsymbol{\alpha} \equiv \nabla \phi$ & gradient of phase field interpreted as the linear density of interfaces \\
\hline$\hat{\boldsymbol{n}} \equiv \frac{\alpha}{|\alpha|}$ & unit normal vector to the interface between phases \\
\hline$\hat{t}$ & unit tangent to a curve in space \\
\hline $\boldsymbol{x}_{0}$ & material particle in the reference configuration \\
\hline $\boldsymbol{x}$ & material particle in the deformed configuration \\
\hline$\nabla \equiv \nabla_{\boldsymbol{x}}$ and $\nabla_{\boldsymbol{x}_{0}}$ & gradient with respect to $\boldsymbol{x}$ and $\boldsymbol{x}_{0}$ respectively; $\nabla_{\boldsymbol{x}}=\boldsymbol{F}^{-T} \nabla_{\boldsymbol{x}_{0}}$ \\
\hline$\Omega$ and $\Omega_{0}$ & the body in the current and reference configuration respectively \\
\hline$\partial \Omega$ and $\partial \Omega_{0}$ & the boundary of $\Omega$ and $\Omega_{0}$ respectively \\
\hline $\boldsymbol{N}$ and $\boldsymbol{N}_{0}$ & the outward normals to $\partial \Omega$ and $\partial \Omega_{0}$ respectively \\
\hline $\boldsymbol{F} \equiv \nabla_{\boldsymbol{x}_{0}} \boldsymbol{x}$ & deformation gradient \\
\hline$\rho_{0}$ & mass density in the reference configuration \\
\hline$J \equiv \operatorname{det} \boldsymbol{F}$ & Jacobian of the deformation \\
\hline $\boldsymbol{C} \equiv \boldsymbol{F}^{T} \boldsymbol{F}$ & Right Cauchy-Green deformation tensor \\
\hline $\boldsymbol{E} \equiv \frac{1}{2}(\boldsymbol{C}-\boldsymbol{I})$ & Green-Lagrangian strain tensor \\
\hline $\boldsymbol{\epsilon} \equiv \frac{1}{2}\left(\boldsymbol{F}+\boldsymbol{F}^{T}\right)-\boldsymbol{I}$ & linearized strain tensor \\
\hline$W(\boldsymbol{F})$ & classical elastic energy density \\
\hline$\left.\stackrel{\circ}{W}^{(\boldsymbol{F}}, \phi\right)$ & modified elastic energy density \\
\hline $\boldsymbol{\sigma}=\frac{\partial W}{\partial \boldsymbol{F}}$ or $\frac{\partial \stackrel{\circ}{W}^{\prime}}{\partial \boldsymbol{F}}$ & First Piola-Kirchhoff stress \\
\hline $\boldsymbol{v}$ & material velocity \\
\hline$v_{n}^{\phi}$ & normal velocity field for interface motion \\
\hline$\hat{v}_{n}^{\phi}$ & kinetic response function for interface normal velocity \\
\hline$G$ & nucleation/source term \\
\hline$f$ & driving force \\
\hline$f_{\text {bulk }}$ & bulk driving force \\
\hline$f_{\text {edge }}$ & edge driving force \\
\hline$\llbracket \cdot \rrbracket$ & The jump in a quantity across an interface \\
\hline
\end{tabular}

For simplicity, we abuse notation and use interchangeably $W(\boldsymbol{F})$ and $W(\boldsymbol{E})$, and ${ }^{\circ}(\boldsymbol{F}, \phi)$ and ${ }^{\circ}(\boldsymbol{E}, \phi)$. For these quantities and $\sigma$, we use the same symbol both for the field and for the material response function.

$H_{l}(x)$ represents a function that resembles the Heaviside step function. It transitions rapidly but smoothly from 0 to 1 and is symmetric about $x=0$, and $l$ represents the scale over which the function transitions. It is assumed to be sufficiently smooth for all derivatives in the paper to be well-defined. The particular choice in this paper is $H_{l}(x)=\frac{1}{2}(1+\tanh (x / l))$. The derivative of $H_{l}(x)$ is written $\delta_{l}(x)$, and is a smooth function that formally approximates the Dirac measure. 


\section{Formulation}

Similar to the standard phase-field models, we use two primary fields, $\boldsymbol{x}$ to describe the deformation, and $\phi$ to track the phase of the material. The evolution of $\boldsymbol{x}$ is governed by balance of linear momentum, i.e. $\operatorname{div}_{\boldsymbol{x}_{0}}\left(\frac{\partial W(\boldsymbol{F}, \phi)}{\partial \boldsymbol{F}}\right)=\rho_{0} \ddot{\boldsymbol{x}}\left(\boldsymbol{x}_{0}, t\right)$. We assume that the elastic energy density $W(\boldsymbol{F})$ and the kinetic and nucleation relations for interfaces have been well-characterized and are available. We aim to formulate a regularized-interface model that has the same elastic response and kinetic and nucleation behavior for interfaces. We describe below how to set up $\stackrel{\circ}{W}(\boldsymbol{F}, \phi)$ given $W(\boldsymbol{F})$, the evolution equation for the kinetics and nucleation of $\phi$, and the thermodynamics associated with our model.

\subsection{Energetics}

We start by assuming that the classical strain energy density $W(\boldsymbol{F})$ of the material is available, perhaps by calibrating to experiment or from lower-scale calculations. We wish to obtain the modified energy density ${ }^{\circ}(\boldsymbol{F}, \phi)$, which will have certain features that provide critical advantages for nucleation, yet stays largely faithful to $W(\boldsymbol{F})$. Therefore, we require the following of $W$ : (i) away from energy barriers, $W(\boldsymbol{F})=\mathscr{W}(\boldsymbol{F}, \phi)$ for the value of $\phi$ that corresponds to the appropriate phase; and (ii) $W^{\circ}$ should be convex in the (linear or nonlinear) strain for a given value of $\phi$, preventing transformations purely through the evolution of $\boldsymbol{F}$, and hence enables the dynamics of $\phi$ to govern the phase transformation.

We begin by considering two phases with characteristic strains given by $\boldsymbol{E}_{1}$ and $\boldsymbol{E}_{2}$. We emphasize that these strains need not correspond to stress-free states, but that the tangent modulus at those points is positive-definite. Then, define the functions for $A=1,2$ :

$$
\psi_{A}(\boldsymbol{E})=W\left(\boldsymbol{E}_{A}\right)+\underbrace{\boldsymbol{\sigma}_{T}}_{\left.\equiv \frac{\partial W}{\partial E}\right|_{\boldsymbol{E}_{A}}}:\left(\boldsymbol{E}-\boldsymbol{E}_{A}\right)+\frac{1}{2}\left(\boldsymbol{E}-\boldsymbol{E}_{A}\right): \underbrace{\boldsymbol{C}_{T}}_{\left.\equiv \frac{\partial^{2} W}{\partial \boldsymbol{E} \partial \boldsymbol{E}}\right|_{\boldsymbol{E}_{A}}}:\left(\boldsymbol{E}-\boldsymbol{E}_{A}\right)
$$

$\psi_{A}$ approximates the behavior of $W$ near the states $\boldsymbol{E}_{1}$ and $\boldsymbol{E}_{2}$, and $\psi_{A}$ are convex in the arguments.

We now define the re-parametrized energy density $\stackrel{\circ}{W}(\boldsymbol{F}, \phi)$ :

$$
\stackrel{\circ}{W}(\boldsymbol{E}, \phi)=\left(1-H_{l}(\phi-0.5)\right) \psi_{1}(\boldsymbol{E})+\left(H_{l}(\phi-0.5)\right) \psi_{2}(\boldsymbol{E})
$$

where $H_{l}$ is a smooth function that resembles the Heaviside (described in Section 1.2). Therefore, $\stackrel{\circ}{W}(\boldsymbol{E}, \phi \simeq 0)=\psi_{1}(\boldsymbol{E})$ and $\stackrel{\circ}{W}(\boldsymbol{E}, \phi \simeq 1)=\psi_{2}(\boldsymbol{E})$; further, $\stackrel{\circ}{W}\left(\boldsymbol{E}_{1}, 0\right)=W\left(\boldsymbol{E}_{1}\right)$ and $\stackrel{\circ}{W}\left(\boldsymbol{E}_{2}, 1\right)=$ $W\left(\boldsymbol{E}_{2}\right)$.

Fig. 1 plots an example of $\mathscr{D}^{W}$ with a scalar strain measure to enable representation on paper. The lowstrain phase corresponds roughly to $0.0<\phi<0.3$, and the high-strain phase corresponds roughly to $0.7<\phi<1.0$. The transition range is roughly $0.3-0.7$. In general, $\phi$ is in the transition range only in the vicinity of an interface. In a uniform phase region, $\phi$ will take on a value appropriate to that phase.

The key reason to re-formulate the energy is to achieve a clear separation between nucleation and kinetics. In standard phase-field models, the form of the energetic coupling between $\phi$ and strain can lead to the nucleation of a new phase in a single-phase region purely through the kinetic equation, making the separation between nucleation and kinetics impossible. Here, $\stackrel{i}{W}$ is independent of $\phi$ if it is outside the transition range; consequently, there can be no driving force for kinetic evolution when $\phi$ is outside this 
range, irrespective of the level of stress or other fields. Consequently, away from an interface, $\phi$ will not evolve through the kinetic response irrespective of the local mechanical state. Hence, the kinetic response cannot cause nucleation of a new phase in a single-phase region. The kinetic equation can play a role only when $\phi$ is in the transition set in the vicinity of an interface, i.e., it can affect the behavior of an interface but not a uniform phase.

While our energy does not permit nucleation, the conservation law that we set up below for interfaces permits us to specify precisely the nature of nucleation. Further, the kinetic equation described there is multiplied by $|\nabla \phi|$, which suppresses the kinetic evolution of $\phi$ when it is spatially-uniform away from an interface. Hence, away from an interface, the only way that $\phi$ can evolve is when the nucleation term - that can be a function of stress or any other field - in the interface conservation law is activated.

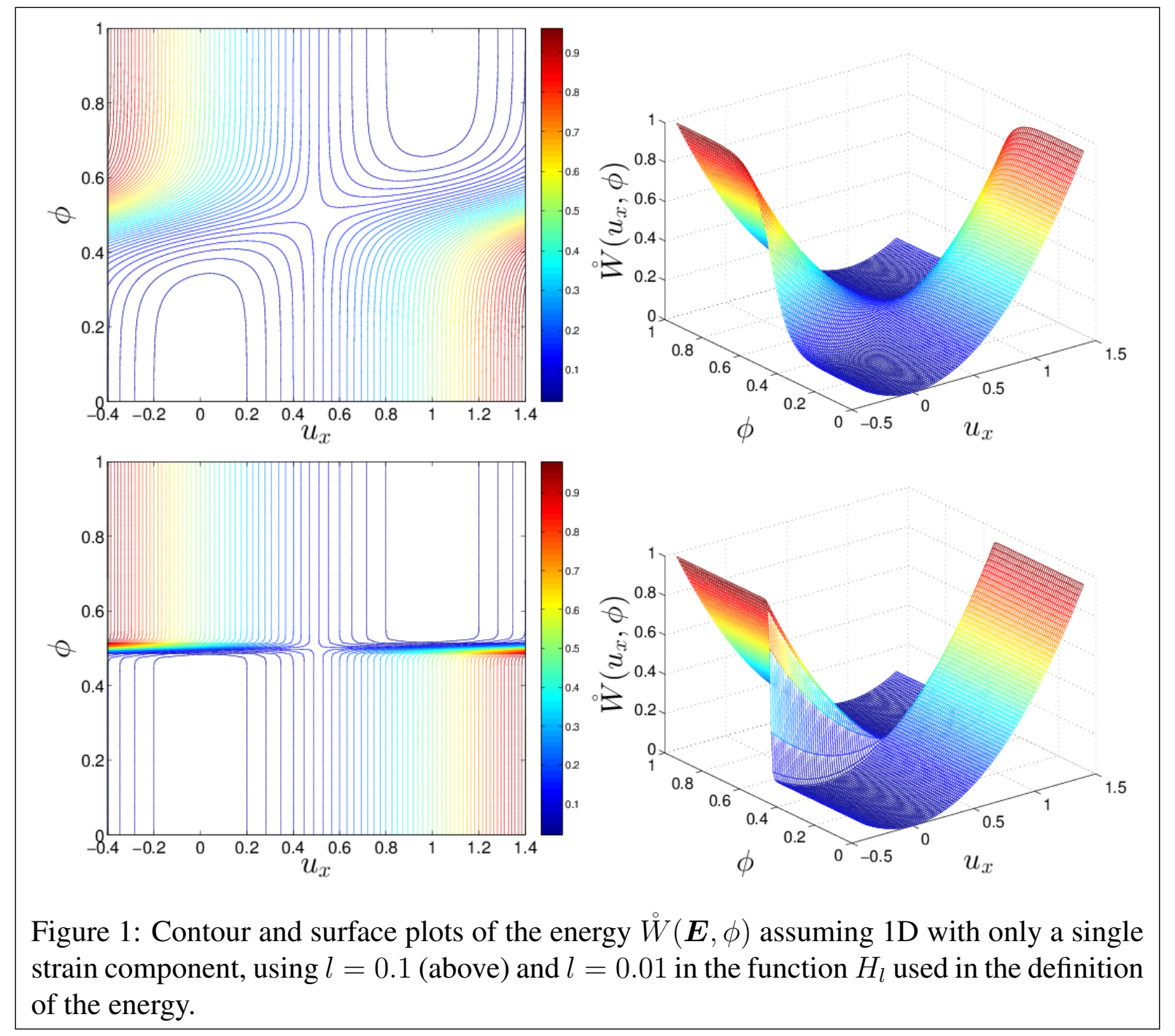

We remark on some features of this energy:

1. Using $\boldsymbol{\sigma}_{T}$, the tangent stress, allows us to position the characteristic strains $\boldsymbol{E}_{1}, \boldsymbol{E}_{2}$ at any point in strain-space where the tangent modulus is positive-definite. These do not need to correspond to stress-free strains, and this property is useful in modeling situations such as stress-induced martensite, where one phase is observed only under stress. 
2. We have used only two terms in the Taylor expansion around the characteristic strains. Increased fidelity to $W$ may be possible with use of additional terms, but this requires care to retain convexity in $\boldsymbol{E}$. Our reason to have convexity is loosely based on obtaining unique solutions, and preventing phase transformations that occur without the evolution of $\phi$. It is possible that convexity can be too strong an assumption [Ant05]. However, this is a larger issue beyond the scope of our work here.

3. $\stackrel{i}{W}$ is faithful to the original energy $W$ near the characteristic strains, but less so further away. At the barriers, it is completely at odds with $W$, because $\stackrel{\circ}{W}$ is convex for fixed $\phi$. However, passage over the barrier is governed by nucleation and kinetics, hence we do not need to accurately model it through $\dot{\circ}^{W}$. We further note that the driving force on an interface in sharp-interface classical elasticity is $f_{\text {class }} \equiv \llbracket W \rrbracket-\langle\boldsymbol{\sigma}\rangle: \llbracket \boldsymbol{F} \rrbracket=W\left(\boldsymbol{F}^{+}\right)-W\left(\boldsymbol{F}^{-}\right)-\frac{1}{2}\left(\boldsymbol{\sigma}\left(\boldsymbol{F}^{+}\right)+\boldsymbol{\sigma}\left(\boldsymbol{F}^{-}\right)\right):\left(\boldsymbol{F}^{+}-\boldsymbol{F}^{-}\right)$, where $\boldsymbol{F}^{ \pm}$are the limiting deformation gradients on either side of the interface [AK06]. Therefore, $f_{\text {class }}$ does not depend on the details of the barrier for given $\boldsymbol{F}^{ \pm}$.

4. Stresses and other applied fields can lead to the usual elastic deformations through the elastic response of each phase in any part of the domain, both near and away from interfaces.

5. The energy density of the body includes a contribution $\frac{1}{2} \epsilon|\nabla \phi|^{2}$. As in standard phase-field models, this prevents the formation of singularly-localized interfaces. Therefore, the total energy written in the reference configuration is $\int_{\Omega_{0}}\left[\stackrel{\circ}{W}(\boldsymbol{F}, \phi)+\frac{1}{2} \epsilon\left|\boldsymbol{F}^{-T} \nabla_{\boldsymbol{x}_{0}} \phi_{0}\right|^{2}\right] d \Omega_{0}$, up to boundary terms. For simplicity, we approximate the gradient contribution in the reference by $\int_{\Omega_{0}} \frac{1}{2} \epsilon\left|\nabla_{\boldsymbol{x}_{0}} \phi_{0}\right|^{2} d \Omega_{0}$.

6. Our energetic prescription shares some features of standard phase-field models. For instance, both models (typically) have convex energy density in the elastic strain for a fixed value of $\phi$. The nonconvexity in $\phi$ in the energy density for standard phase-field models is observed when the strain is allowed to completely relax to the stress-free state for each value of $\phi$. Our energy also has this nonconvexity, which can be seen in examining Fig. 1: if we increase $\phi$ while traversing a curve that minimizes the energy with respect to the strain, we see that it is nonconvex. While it is possible that one would not call them "wells", because the energy remains constant when $\phi$ is outside the critical range, there is nonconvexity in our model; for example, the point $(0.5,0.5)$ in the strain- $\phi$ space is a barrier between the low-energy states.

\subsection{Evolution Law}

Our starting point in formulating the evolution of $\phi$ is to note that $\nabla \phi$ provides, roughly, a measure of the number or "strength" of the interfaces in the $\phi$ field per unit length, Fig. 2.

In general, given a field $\phi(\boldsymbol{x})$ with localized transitions between constant values, we can readily locate the interfaces in this field using $\nabla \phi$. Further, if we pick any curve and integrate $\nabla \phi$ along this curve, the value that we obtain provides a measure of the net number of interfaces that we have traversed, assuming that all interfaces have the same "strength". If the interfaces have different strengths, we obtain a measure of the net interface strength that we have traversed. This physical picture provides the intuition behind what follows, but it also expresses the simple fact that if we have a single-valued field $\phi$, then integrating the gradient is simply the difference between $\phi$ at either end of the curve.

The geometric picture is roughly related to gradients of fields being so-called 1-forms, i.e., they are objects that are naturally integrated along curves [MH94]. Analogies of this are commonplace in elasticity: e.g., the divergence of a field is a 3-form and is naturally integrated over volumes, as is used in 


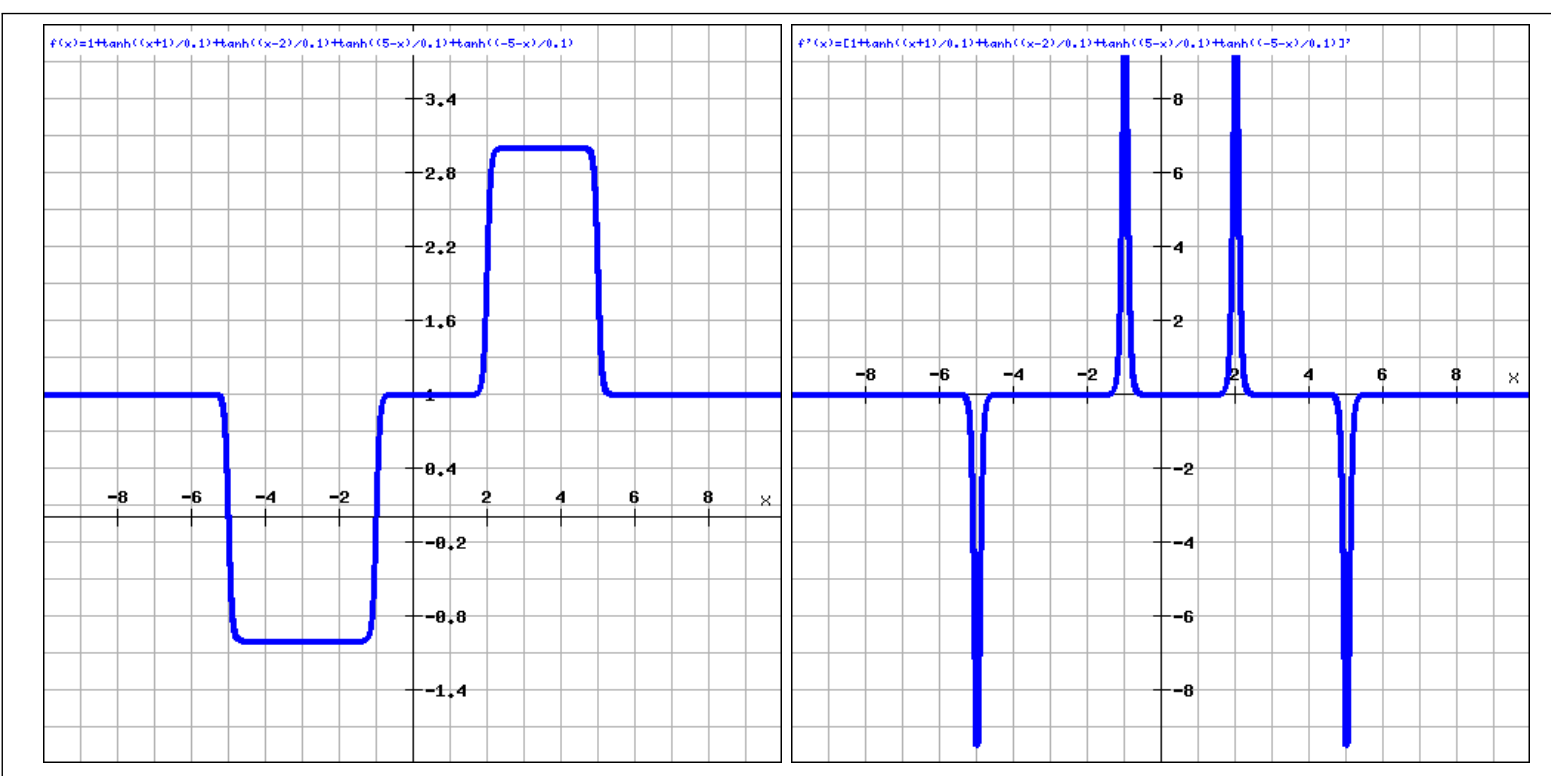

Figure 2: Left: a field $\phi$ with a number of interfaces. Right: $\nabla \phi$ provides a measure of (signed) interface density per unit length.

the conservation laws for mass, momentum, and energy. The curl of a field is a 2-form and is naturally integrated over surfaces, as is used in proving the single-valuedness of a deformation field corresponding to curl $\boldsymbol{F}=\mathbf{0}$, as well as in dislocation mechanics where curl $\boldsymbol{F}$ provides an areal density of dislocation line defects [Ach01].

Given this notion of the interface density field $\nabla \phi$, we then formulate a balance law (see Fig. 3). Let the interfaces have a normal velocity given by the field $v_{n}^{\phi}$; note that this velocity is distinct from the material velocity $\dot{\boldsymbol{u}}$. Now consider a curve $C(t)$ in space. This curve "threads" or passes through some number of interfaces. Further, interfaces are entering or exiting at both ends of the curve due to their motion described by the field $v_{n}^{\phi}$. The conservation principle is that the net increase in the number of interfaces that are threaded by $C(t)$ is a balance between interfaces entering, interfaces exiting, and interfaces being created and destroyed by sources and sinks.

$$
\frac{d}{d t}\left\{\begin{array}{c}
\text { interfaces threaded } \\
\text { by the curve }
\end{array}\right\}=\left\{\begin{array}{c}
\text { interfaces entering } \\
\text { the curve }
\end{array}-\begin{array}{c}
\text { interfaces leaving } \\
\text { the curve }
\end{array}\right\}+\left\{\begin{array}{c}
\text { interface } \\
\text { creation }
\end{array}\right\}
$$

Using that this must hold for every curve $C(t)$ enables us to localize the balance law.

The flux of interfaces through the ends of the curve $C(t)$ can be computed by referring to Fig. 3 . Let $\hat{\boldsymbol{t}}$ be the unit tangent to the end of the curve, and $\hat{n} \equiv \frac{\nabla \phi}{|\nabla \phi|}$ the unit normal to the interface. Then the flux can be written

$$
|\nabla \phi| \frac{\nabla \phi \cdot \hat{\boldsymbol{t}}}{|\nabla \phi \cdot \hat{\boldsymbol{t}}|} \frac{v_{n}^{\phi}}{\hat{\boldsymbol{n}} \cdot \hat{\boldsymbol{t}}}|\hat{\boldsymbol{t}} \cdot \hat{\boldsymbol{n}}|
$$

The first term represents the strength of the interface; the second term is simply +1 if the interface enters and -1 if it leaves; the third term is the velocity of the interface projected onto the $\hat{t}$ direction to obtain the velocity relative to the curve direction, i.e. the distance along the $\hat{t}$ direction traversed by the interface in unit time; and the fourth term picks out only the portion of the flux that is threading the curve by moving along $\hat{t}$. 


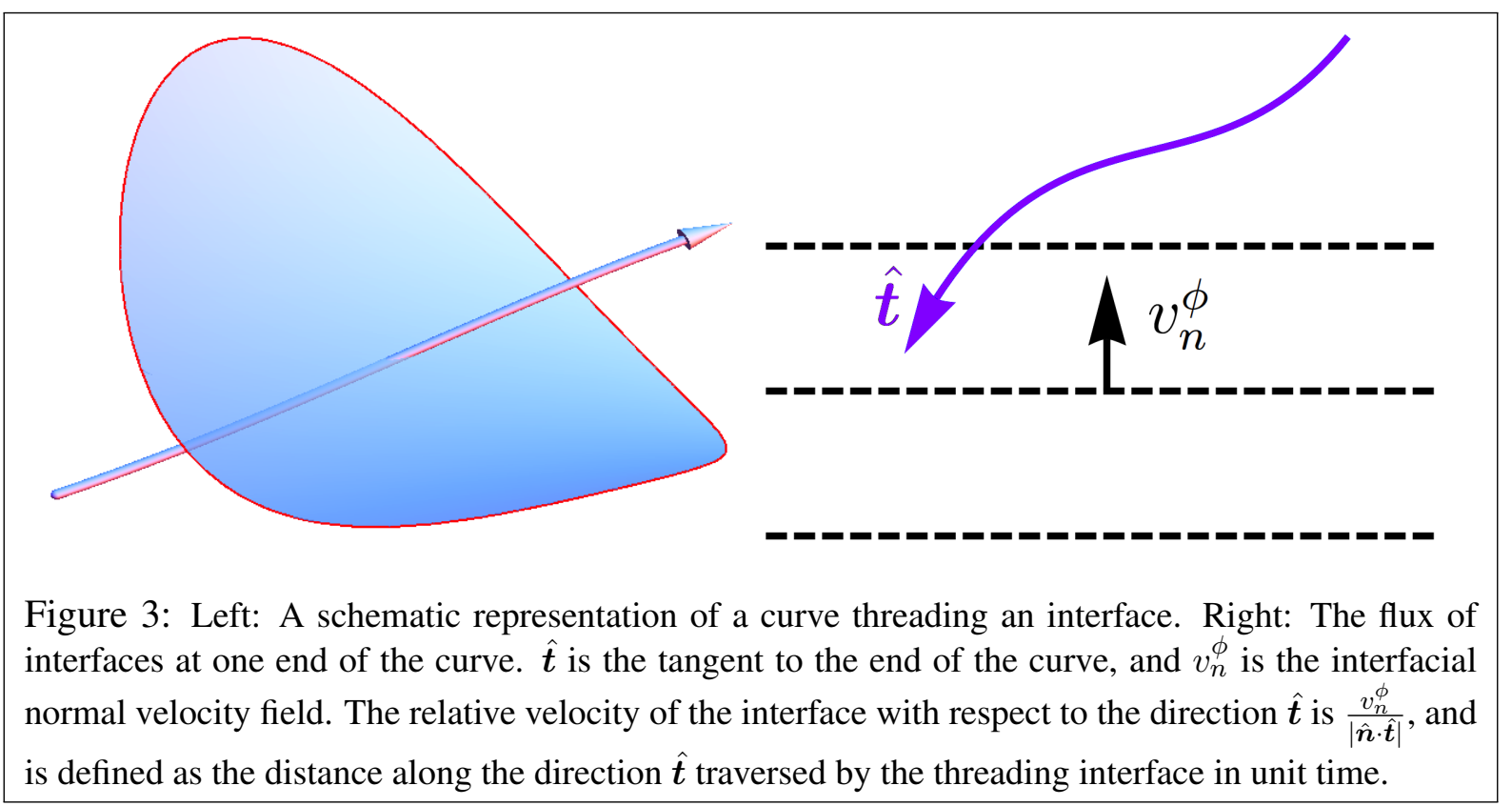

An alternate picture is to note that $\nabla \phi \cdot \hat{\boldsymbol{t}}$ is the (signed) interface density along the direction $\hat{\boldsymbol{t}}$, and $\frac{v_{n}^{\phi}}{\hat{\boldsymbol{n}} \cdot \hat{\boldsymbol{t}}}$ is the velocity of the interface relative to the direction $\hat{t}$, so the flux is simply:

$$
\nabla \phi \cdot \hat{\boldsymbol{t}} \frac{v_{n}^{\phi}}{\hat{\boldsymbol{n}} \cdot \hat{\boldsymbol{t}}}
$$

Both expressions for the flux are identical, and simplify to $|\nabla \phi| v_{n}$ when we substitute $\hat{\boldsymbol{n}} \equiv \frac{\nabla \phi}{|\nabla \phi|} 2$.

Defining the interface density $\boldsymbol{\alpha}:=\nabla \phi$, we have:

$$
\underbrace{\left.|\boldsymbol{\alpha}| v_{n}^{\phi}\right|_{C^{-}} ^{C^{+}}}_{\text {net flux of interfaces }}=\underbrace{\frac{d}{d t} \int_{C(t)} \boldsymbol{\alpha} d \boldsymbol{x}}_{\text {increase in number of interfaces threaded }}-\underbrace{\int_{C(t)} \boldsymbol{S} d \boldsymbol{x}}_{\text {source of new interfaces }}
$$

We can transform $\left.|\boldsymbol{\alpha}| v_{n}^{\phi}\right|_{C^{-}} ^{C^{+}}=\int_{C(t)} \nabla\left(|\boldsymbol{\alpha}| v_{n}^{\phi}\right) d \boldsymbol{x}$.

Using that $C(t)$ is a material curve, we can write the mapping $d \boldsymbol{x}=\boldsymbol{F} d \boldsymbol{x}_{0}$ between the infinitesimal elements of $C(t)$ and its image $C_{0}$ in the reference. Then, the time derivative can be transformed to $\frac{d}{d t} \int_{C_{0}} \boldsymbol{\alpha} \boldsymbol{F} d \boldsymbol{X}=\int_{C(t)}(\dot{\boldsymbol{\alpha}}+\boldsymbol{\alpha} \boldsymbol{L}) d \boldsymbol{x}$ where $\boldsymbol{L}$ is the spatial velocity gradient.

This lets us localize to obtain:

$$
\dot{\boldsymbol{\alpha}}=\nabla\left(|\boldsymbol{\alpha}| v_{n}^{\phi}\right)+\boldsymbol{S}(\boldsymbol{x}, t)-\boldsymbol{\alpha} \boldsymbol{L}
$$

Noting that the source is constrained by the above equation to be of the form, i.e. $\boldsymbol{S}=\nabla G+\boldsymbol{\alpha} \boldsymbol{L}$, we can integrate the above equation to obtain:

$$
|\nabla \phi| v_{n}^{\phi}+G=\dot{\phi}
$$

\footnotetext{
${ }^{2}$ A. Acharya gave a different argument for why the flux must have this final form that guided us, and also many useful discussions on Section 2.2.
} 
The nucleation / source term $G$ can be an arbitrary function of any of the fields in the problem, up to some weak limitations imposed by thermodynamics (discussed below). The term $S$ must be a gradient up to the term $\boldsymbol{\alpha} \boldsymbol{L}$, and represents the fact that in a single-valued field $\phi$, interfaces that nucleate must either terminate on the boundary or close on themselves but cannot end in the interior of the body.

We note certain important features of the evolution law that we have posed:

1. The kinetics of existing interfaces is constitutively prescribed through the interface velocity field $v_{n}^{\phi}$, which can be a function of stress, strain, as well as any other relevant quantity, such as the work-conjugate to $\phi$ (the Eshelby / configurational force). This makes it trivial to obtain complex kinetics; for instance, if the interface is pinned below a critical value of the stress, we simply prescribe that $v_{n}^{\phi}$ is zero at all spatial points where the stress is below the critical value. Similarly, other kinds of nonlinear and complex kinetics can be readily incorporated.

2. Nucleation of new interfaces is prescribed through the source term in the balance law, and provides precise control on the nucleation process. For instance, we can prescribe that a source is activated only beyond some critical stress and stress rate; thus, for example, it is straightforward to model a nucleation process in which the critical nucleation stress is extremely sensitive to strain rate. In addition, the activation of the source can be completely heterogeneous and vary vastly from point to point.

3. The appearance of $|\nabla \phi|$ in the evolution is important to separate kinetics from nucleation: if we have a large driving force in a uniform phase far away from an interface, $|\nabla \phi|$ will remain 0 and therefore will not allow the kinetic term to play a role irrespective of driving force, stress, etc.

We note that an analogous idea to the conservation principle stated above is used in [Ach01] to obtain an evolution law for dislocations, and recently in [AD14, PAD14] for disclination dynamics. In [Ach01], by connecting the dislocation density to curl $\boldsymbol{F}$ and using the physical picture that these are line defects, a conservation law is posed by using that the rate of change of dislocations intersecting an arbitrary area element is related to the net flux of intersecting dislocations and the creation of intersecting dislocations. The conservation law that we have posed in this work builds on this picture, and the key point of departure of our work is the idea that the balance principle from [Ach01] can be extended from line defects detected by curl $\cdot$ to interfacial defects that are detected by $\nabla \cdot$. A further use of this approach are the standard continuum balances of mass, momentum, energy that are all posed with volumetric densities, and the appropriate quantity to be integrated over a volume is div $\cdot$.

In Appendix A, we examine the relation between this conservation principle and Noether's theorem.

\subsubsection{Balance Law in the Reference Configuration}

The entire argument above was posed in the current configuration. Since the field $\phi$ relates to the state of material particles, it would be physically reasonable to alternatively pose the balance principle in the reference configuration. We examine this approach briefly.

In this section, quantities with subscripts of 0 denote referential objects. $\boldsymbol{x}_{0}$ is the referential pre-image of the material particle $\boldsymbol{x}\left(\boldsymbol{x}_{0}, t\right)$. We make the natural transformation that $\phi$ is the same in the reference and the current for a given material particle at a given time: $\phi_{0}\left(\boldsymbol{x}_{0}(\boldsymbol{x}, t), t\right)=\phi(\boldsymbol{x}, t)$. From standard manipulations of continuum mechanics, it follows that $\boldsymbol{\alpha} \boldsymbol{L}+\dot{\boldsymbol{\alpha}}=\boldsymbol{F}^{-T} \dot{\boldsymbol{\alpha}}_{0}$. We further make the identification that $\boldsymbol{S}=\boldsymbol{F}^{-T} \boldsymbol{S}_{0} \Leftrightarrow G=G_{0}$. 
Substituting in the balance principle (2.6), we can write:

$$
\boldsymbol{F}^{-T} \dot{\boldsymbol{\alpha}}_{0}=\boldsymbol{F}^{-T} \boldsymbol{S}_{0}+\boldsymbol{F}^{-T} \nabla_{\boldsymbol{x}_{0}}\left(|\boldsymbol{\alpha}| v_{n}^{\phi}\right)
$$

We have also used above that $\nabla_{\boldsymbol{x}}=\boldsymbol{F}^{-T} \nabla_{\boldsymbol{x}_{0}}$.

The natural transformation induced on the interface velocity field is obtained by requiring $|\boldsymbol{\alpha}| v_{n}^{\phi}=$ $\left|\alpha_{0}\right| v_{n 0}^{\phi}$. The result is the non-standard transformation $v_{n 0}^{\phi} \hat{\boldsymbol{n}}_{0}=\boldsymbol{F}^{-1} v_{n}^{\phi} \hat{\boldsymbol{n}}$. This is deceptively simple, because the transformation between $\hat{\boldsymbol{n}}_{0}$ to $\hat{\boldsymbol{n}} \equiv \nabla \phi /|\nabla \phi|$ is not as a standard normal to a material surface. The final result can be compactly written $v_{n 0}^{\phi}=v_{n}^{\phi}\left(\hat{\boldsymbol{n}}_{0} \boldsymbol{F}^{-1} \boldsymbol{F}^{-T} \hat{\boldsymbol{n}}_{0}\right)^{\frac{1}{2}}$.

Using this further transformation of $v_{n}^{\phi}$, we obtain the interface balance in the reference configuration:

$$
\dot{\boldsymbol{\alpha}}_{0}=\boldsymbol{S}_{0}+\nabla_{\boldsymbol{x}_{0}}\left(\left|\boldsymbol{\alpha}_{0}\right| v_{n 0}^{\phi}\right)
$$

This can be readily integrated once to obtain

$$
\dot{\phi}_{0}=G_{0}+\left|\boldsymbol{\alpha}_{0}\right| v_{n 0}^{\phi}
$$

There are two practical, though minor, advantages to the referential form of the balance principle. First, frame-indifference is readily seen to be satisfied. Second, the interpretation of $\boldsymbol{S}_{0}=\nabla_{\boldsymbol{x}_{0}} G_{0}$ is simpler without the additional terms from the material derivative.

\subsection{Thermodynamics and Dissipation}

Following established ideas, we use the statement of the second law that the dissipation must be nonnegative for every motion of the body to find the thermodynamic conjugate driving forces for kinetics and nucleation. The dissipation is defined as the deficit between the rate of external work done and the increase in stored energy:

$$
\mathcal{D}=\text { External working }-\frac{d}{d t}\left(\int_{\Omega_{0}}\left[\stackrel{\circ}{W}(\boldsymbol{F}, \phi)+\frac{1}{2} \epsilon \frac{\partial \phi_{0}}{\partial x_{0 i}} \frac{\partial \phi_{0}}{\partial x_{0 i}}\right] d \Omega_{0}+\frac{1}{2} \int_{\Omega_{0}} \rho_{0} V_{0 i} V_{0 i} d \Omega_{0}\right)
$$

This can be manipulated to find the conjugates to $v_{n}^{\phi}$ and $G$.

$$
\mathcal{D}=\text { External working }-\int_{\Omega_{0}}\left[\frac{\partial \stackrel{\circ}{\partial}}{\partial F_{i j}} \frac{d F_{i j}}{d t}+\frac{\partial \stackrel{\circ}{\partial}}{\partial \phi} \frac{d \phi}{d t}+\epsilon \frac{\partial \phi_{0}}{\partial x_{0 i}} \frac{d}{d t} \frac{\partial \phi_{0}}{\partial x_{0 i}}\right] d \Omega_{0}-\int_{\Omega_{0}} \rho_{0} V_{0 i} \dot{V}_{0 i} d \Omega_{0}
$$

Using $\frac{\partial F_{i j}}{\partial t}=\frac{\partial V_{0 i}}{\partial x_{0 j}}$ and integration-by-parts:

$$
\begin{aligned}
\mathcal{D}= & \text { External working }-\int_{\Omega_{0}} \frac{\partial}{\partial x_{0 j}}\left(\frac{\partial \stackrel{\circ}{\partial}}{\partial F_{i j}} V_{0 i}\right) d \Omega_{0}+\int_{\Omega_{0}} V_{0 i}\left(\frac{\partial}{\partial x_{0 j}} \frac{\partial \stackrel{\circ}{W}^{\prime}}{\partial F_{i j}}-\rho_{0} \dot{V}_{0 i}\right) d \Omega_{0} \\
& -\int_{\Omega_{0}}\left[\frac{\partial \stackrel{W}{\partial \phi}}{\partial \phi}+\epsilon \frac{\partial \phi_{0}}{\partial x_{0 i}} \frac{d}{d t} \frac{\partial \phi_{0}}{\partial x_{0 i}}\right] d \Omega_{0}
\end{aligned}
$$


The first integral above is exactly balanced by the external work done by boundary tractions ${ }^{3}$. The second integral is identically zero from balance of linear momentum. Therefore, the dissipation simplifies to:

$$
\mathcal{D}=\int_{\Omega_{0}}\left[-\frac{\partial \stackrel{\circ}{\partial}}{\partial \phi}+\epsilon \frac{\partial^{2} \phi_{0}}{\partial x_{0 i} \partial x_{0 i}}\right] \frac{d \phi_{0}}{d t} d \Omega_{0}-\int_{\partial \Omega_{0}} \epsilon \frac{\partial \phi_{0}}{\partial x_{0 i}} N_{0 i} \frac{d \phi}{d t} d \partial \Omega_{0}
$$

We continue to assume that there is no dissipation at the boundary, and hence use the boundary condition $\nabla_{x_{0}} \phi_{0} \cdot \boldsymbol{N}_{0}=0$. This also corresponds to the standard boundary condition used in phase-field models. In those models, taking the variation of the energy and moving terms to the boundary leads to $\nabla \phi \cdot \boldsymbol{n}=0$ when there is no working on $\phi$ at the boundary. Physically, it implies a boundary kinetics that corresponds to the interface configuring itself instantaneously to always keep the boundary driving force zero, thereby not providing a dissipative mechanism. This can be noted from the expression for the driving force for the interface junction with boundary, $f_{\text {edge }}$, in the companion paper (Section on boundary kinetics); setting the terms $t$ and $\epsilon_{S}$ to 0 there recovers the current model, and $f_{\text {edge }}$ is precisely $\nabla \phi \cdot \boldsymbol{n}$.

We substitute the balance law $\dot{\phi}_{0}=G_{0}+\left|\boldsymbol{\alpha}_{0}\right| v_{n 0}^{\phi}$, to get:

$$
\mathcal{D}=\int_{\Omega_{0}}\left[-\frac{\partial \stackrel{\circ}{\partial}}{\partial \phi}+\epsilon \frac{\partial^{2} \phi_{0}}{\partial x_{0 i} \partial x_{0 i}}\right]\left(G_{0}+\left|\nabla_{\boldsymbol{x}_{0}} \phi\right| v_{n 0}^{\phi}\right) d \Omega_{0}
$$

Defining the driving force $f:=-\left[-\frac{\partial \stackrel{\circ}{\partial \phi}}{\partial \phi}+\epsilon \frac{\partial^{2} \phi_{0}}{\partial x_{0 i} \partial x_{0 i}}\right]$, we get:

$$
\mathcal{D}=\int_{\Omega_{0}} f\left(G_{0}+\left|\nabla_{\boldsymbol{x}_{0}} \phi\right| v_{n 0}^{\phi}\right) d \Omega_{0}
$$

To ensure that dissipation is always non-negative, we need both $f G_{0}$ and $f\left|\nabla_{\boldsymbol{x}_{0}} \phi\right| v_{n 0}^{\phi}$ to be non-negative. These are fairly easy conditions to satisfy in a material model. For kinetics, we choose the constitutive response of the form $v_{n 0}^{\phi}=\frac{f}{|f|} \hat{v}_{n 0}^{\phi}(|f|, \ldots)$, where the constitutive response function $\hat{v}_{n 0}^{\phi}$ can be any nonnegative function of the arguments, and the list of arguments can consist of any of the field variables, as well as possibly nonlocal quantities. A similarly weak requirement holds for nucleation.

\subsection{Formal Sharp-Interface Limit}

We consider briefly the formal limit of the driving force in the sharp-interface limit $\epsilon=0$. We emphasize that this is not rigorous, as the limit $\epsilon \rightarrow 0$ involves the delicate singular perturbation of a nonlinear hyperbolic equation.

We start with the dissipation expression ignoring the nucleation contribution:

$$
\mathcal{D}=\int_{\Omega_{0}}-\frac{\partial \stackrel{\circ}{W}}{\partial \phi}\left|\nabla_{\boldsymbol{x}_{0}} \phi\right| v_{n 0}^{\phi} d \Omega_{0}=\int_{\Omega_{0}}-\frac{\partial \stackrel{\circ}{W}}{\partial \phi} \nabla_{\boldsymbol{x}_{0}} \phi \underbrace{\left(\frac{v_{n 0}^{\phi} \nabla_{\boldsymbol{x}_{0}} \phi}{\left|\nabla_{\boldsymbol{x}_{0}} \phi\right|}\right)}_{=: \boldsymbol{v}_{n 0}^{\phi}} d \Omega_{0}
$$

\footnotetext{
${ }^{3}$ We assume that there is no work done on $\phi$ at the boundary for now. We revisit this in the section on boundary kinetics in the companion paper.
} 


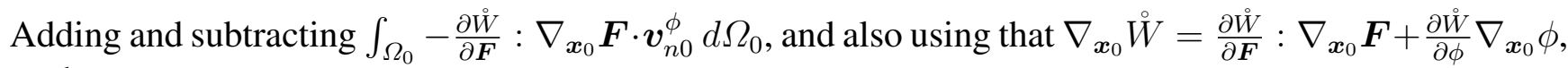
we have

$$
\mathcal{D}=-\int_{\Omega_{0}} \nabla_{\boldsymbol{x}_{0}} \stackrel{\circ}{W} \cdot \boldsymbol{v}_{n 0}^{\phi} d \Omega_{0}+\int_{\Omega_{0}} \frac{\partial \stackrel{\circ}{W}}{\partial \boldsymbol{F}}: \nabla_{\boldsymbol{x}_{0}} \boldsymbol{F} \cdot \boldsymbol{v}_{n 0}^{\phi} d \Omega_{0}
$$

We now further assume the following: (i) the evolution is quasistatic, i.e. inertia is negligible; (ii) the phase boundary is flat and the fields are one-dimensional; and (iii) $\boldsymbol{v}_{n 0}^{\phi}$ is constant in space. The assump-

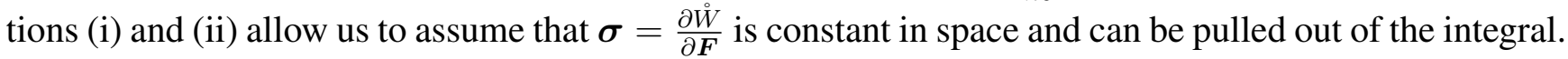
Assumption (iii) is a direct consequence of assuming steady motion of the phase boundary as a traveling wave (Section 3), and allows us to pull $\boldsymbol{v}_{n 0}^{\phi}$ out of the integral.

With these assumptions, we can write:

$$
\mathcal{D}=-(\underbrace{\int_{\Omega_{0}} \nabla_{\boldsymbol{x}_{0}} \stackrel{\circ}{W} d \Omega_{0}}_{\llbracket W \cap \hat{\boldsymbol{n}}}-\boldsymbol{\sigma}: \underbrace{\int_{\Omega_{0}} \nabla_{\boldsymbol{x}_{0}} \boldsymbol{F} d \Omega_{0}}_{\llbracket \boldsymbol{F} \rrbracket \otimes \hat{\boldsymbol{n}}}) \cdot \boldsymbol{v}_{n 0}^{\phi}
$$

which is identical to the driving force obtained by Abeyaratne and Knowles [AK06] in the quasistatic setting.

Given that we recover the classical driving force under these assumptions, it is reasonable to further expect that key properties of the classical theory, e.g. the Maxwell stress at which the driving force vanishes in quasistatics, are also captured correctly.

\section{Traveling Waves in One Dimension}

We investigate the behavior of traveling wave solutions in our model. These correspond to steadily moving interfaces.

For simplicity, we use a one-dimensional setting with linearized kinematics. For $\stackrel{W}{W}$, we use the form:

$$
\stackrel{\circ}{W}\left(u_{x}, \phi\right)=\left(1-H_{l}(\phi-0.5)\right) \frac{1}{2} C\left(u_{x}-\varepsilon_{1}\right)^{2}+H_{l}(\phi-0.5) \frac{1}{2} C\left(u_{x}-\varepsilon_{2}\right)^{2}
$$

We use $\varepsilon_{1}=0$ and $\varepsilon_{2}=1$. The stress is $\sigma=\frac{\partial \stackrel{\circ}{W}}{\partial\left(u_{x}\right)}=C\left(u_{x}-H_{l}(\phi-0.5)\right)$, and the driving force is $f=\delta_{l}(\phi-0.5) \cdot\left(u_{x}-0.5\right)+\epsilon \phi_{x x}$.

We search for traveling wave solutions of the form $u(x, t)=U(x-V t)$ and $\phi(x, t)=\Phi(x-V t)$ for a few different given kinetic relations. We substitute these into the balance of linear momentum and the evolution equation. Below, $U^{\prime}$ and $\Phi^{\prime}$ denote derivatives of $U$ and $\Phi$. We assume that the kinetic response $\hat{v}_{n}^{\phi}(\ldots)$ is a function of only the driving force $f$, and further that it is a monotone function and hence invertible.

From momentum balance, we obtain:

$$
\begin{gathered}
\rho u_{t t}=\sigma_{x} \Rightarrow V^{2} \rho U^{\prime \prime}=C\left\{U^{\prime \prime}-\delta_{l}(\Phi-0.5) \Phi^{\prime}\right\} \Rightarrow\left(1-M^{2}\right) U^{\prime \prime}=\delta_{l}(\Phi-0.5) \Phi^{\prime} \\
\Rightarrow U^{\prime}=\frac{H_{l}(\Phi-0.5)+\tilde{c}_{2}}{1-M^{2}}=\frac{H_{l}(\Phi-0.5)}{1-M^{2}}+c_{2}
\end{gathered}
$$


where $c_{2} \equiv \frac{\tilde{c}_{2}}{1-M^{2}}$ is a constant of integration, and $M$ is the Mach number. We see that as $M \rightarrow 1$, the derivative is unbounded unless $H_{l}(\phi-0.5)+\tilde{c}_{2}=0$. The latter condition requires that $\Phi$ is constant in space, implying that only elastic waves and not phase interfaces are permitted at $M=1$. As expected, this limitation is a consequence of momentum balance alone.

Next, from the evolution equation, we obtain:

$$
\dot{\phi}=\left|\phi_{x}\right| \hat{v}_{n}^{\phi}(|f|) \Rightarrow-V \Phi^{\prime}=\left|\Phi^{\prime}\right| v_{n}^{\phi}
$$

(3.3) implies that the interface velocity field $v_{n}^{\phi}$ is constant in space and time.

Using further that the kinetic response is a monotone function of $f$ implies that the driving force field has to be a constant in space and time. Therefore, $f=\delta_{l}(\Phi-0.5) \cdot\left(U^{\prime}-0.5\right)+\epsilon \Phi^{\prime \prime}=$ const., and substituting for $u^{\prime}$ from (3.2) gives an ODE in $\Phi$ :

$$
f=\text { const. }=\epsilon \Phi^{\prime \prime}+\delta_{l}(\Phi-0.5) \cdot\left(\frac{H_{l}(\Phi-0.5)}{1-M^{2}}-\frac{1}{2}-c_{2}\right)
$$

Given a value of $M$ or alternatively $V$, we can solve this equation to obtain $\Phi$. Also, given $M$, the value of $f$ is obtained from the assumed kinetic response.

(3.4) is a nonlinear ODE because of $H_{l}(\Phi-0.5)$ and $\delta_{l}(\Phi-0.5)$. So we seek to find approximate solutions numerically using finite differences and least-squares minimization following [DB06]. Divide the domain of length $L=1$ into $N$ elements each of length $\Delta x=L / N$; the $N+1$ grid points are denoted $x_{i}$. Discretize the ODE with as:

$$
g\left(x_{i}\right):=\epsilon \frac{\Phi\left(x_{i+1}\right)-2 \Phi\left(x_{i}\right)+\Phi\left(x_{i-1}\right)}{(\Delta x)^{2}}+\delta_{l}\left(\Phi\left(x_{i}\right)-0.5\right) \cdot\left(\frac{H_{l}\left(\Phi\left(x_{i}\right)-0.5\right)}{1-M^{2}}-\frac{1}{2}-c_{2}\right)-f
$$

Define the residue $\mathcal{R}:=\sum_{i=2}^{N}\left|g\left(x_{i}\right)\right|^{2}$. To find $\Phi$, we minimize $\mathcal{R}$ with respect to the nodal values $\Phi_{i}:=$ $\Phi\left(x_{i}\right)$; at the completion of minimization, we evaluate $\mathcal{R}$ to ensure that it is near 0 and we have not found a local minimum. To prevent the solution algorithm from finding trivial single-phase solutions, we fix $\left.\Phi\right|_{x=0.5}=0.5$.

We note that there is an additional constant $c_{2}$ that is unknown. From classical sharp-interface analyses, we expect that the combination of momentum balance and kinetic relation should give us a unique solution in (3.4) once $M$ is fixed. Examining (3.2), we can infer that it is related to the strains / stresses at $\pm \infty$, but it is not clear how exactly to find this explicitly. Therefore, we simply treat $c_{2}$ as an additional variable over which to minimize $\mathcal{R}$.

Fig. 4 plots the solutions for $U$ and $\Phi$ for a linear kinetic relation. Qualitatively similar profiles are obtained for a quadratic kinetic relation.

We extract $\left(U^{\prime}\right)^{ \pm}$, the limiting constant strains far from the interface, and use these to evaluate the classi-

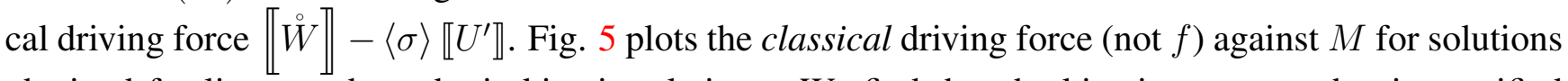
obtained for linear and quadratic kinetic relations. We find that the kinetic response that is specified through the response function $\hat{v}_{n}^{\phi}$ is reproduced in terms of the classical driving force. This supports the belief that our model provides the advantages of both the sharp-interface and the regularized-interface models without the disadvantages of either. We note that the classical kinetic relation deviates from the kinetic response function as $M \rightarrow 1$, but this is expected from linear momentum balance. 

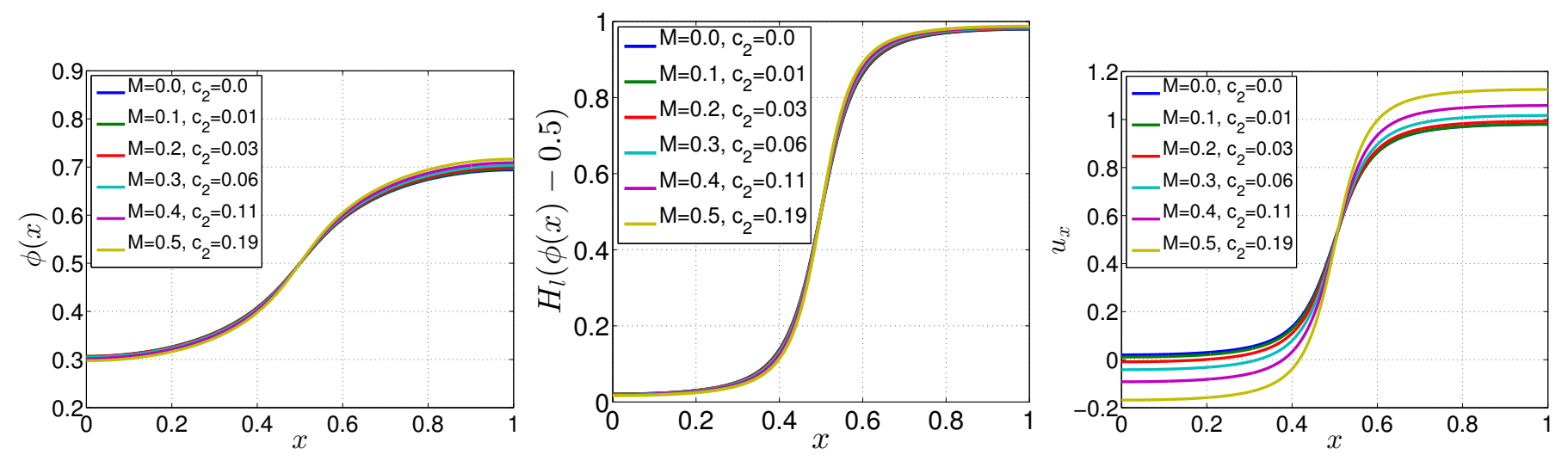

Figure 4: Plots of $\Phi, H_{l}(\Phi-0.5), U^{\prime}$ respectively for traveling wave solutions with different values of $M$ using linear kinetics.

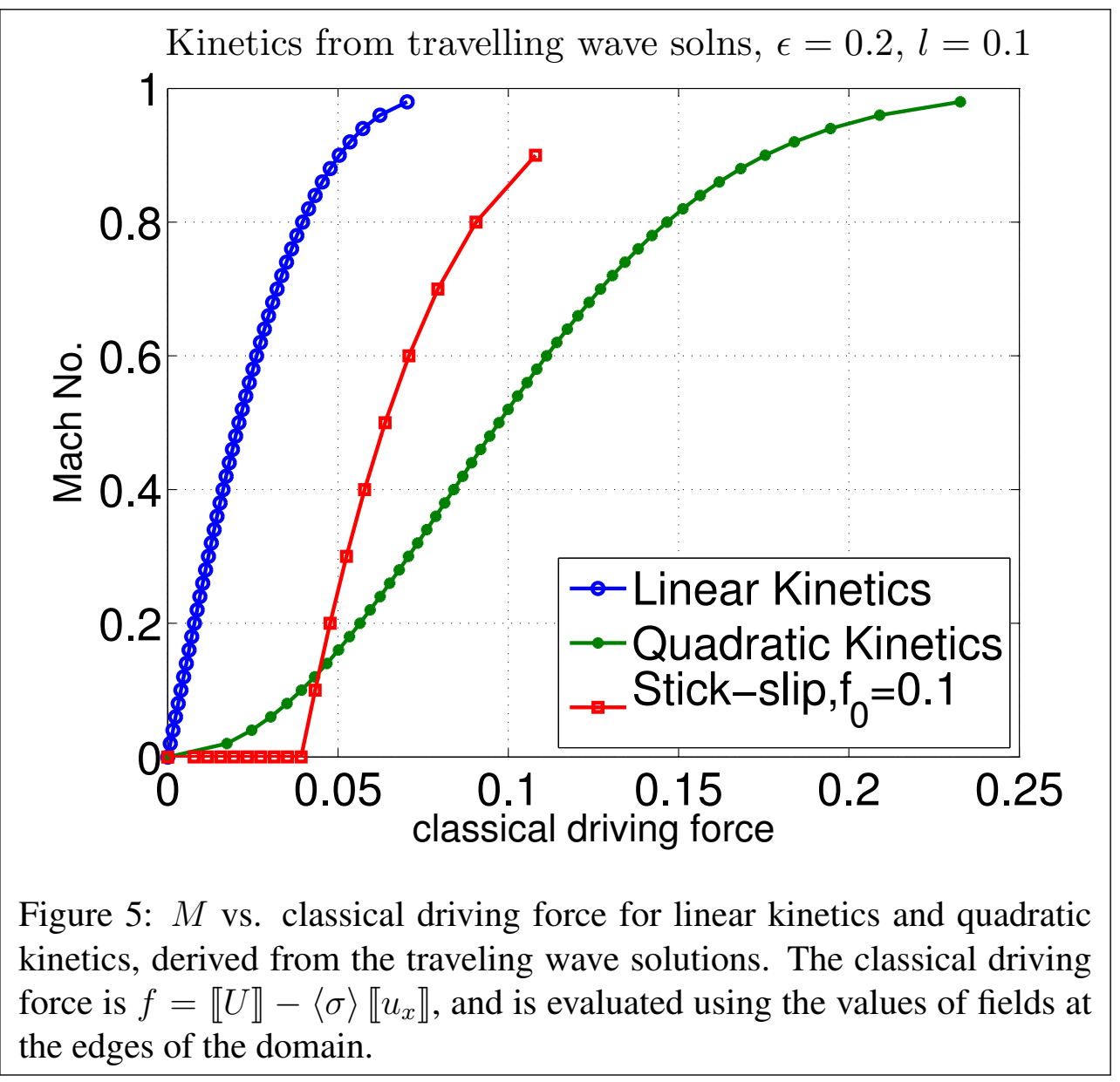

We emphasize an interesting difference between our model and existing phase-field models. In our model, the driving force field and the interface velocity field are both constant in space. Therefore, the relation between them is a simple relation between two scalar quantities, and the notion of a kinetic relation is well-defined. In existing phase-field models, the driving force field is large near an interface and goes to zero away from the interface, i.e. it is a function of location. Therefore, there is no obvious unique scalar 
measure of the driving force that one can extract from this field; one could use the maximum value, or the mean value in some region, and so on. In this perspective, our model has the advantage that it has a closer link to the classical continuum model because there is a unique and obvious relation between driving force and interface velocity.

\section{Dynamics of Interfaces in One Dimension}

We examine the kinetics of phase interfaces through direct dynamic simulations. We solve linear momentum balance along with the evolution equation for $\phi$ in various configurations and with various choices for the kinetic response.

We use the following material model:

$$
\begin{aligned}
& \stackrel{\circ}{W}\left(u_{x}, \phi\right)=\left(1-H_{l}(\phi-0.5)\right) \frac{1}{2} C\left(u_{x}-\varepsilon_{1}\right)^{2}+H_{l}(\phi-0.5) \frac{1}{2} C\left(u_{x}-\varepsilon_{2}\right)^{2}
\end{aligned}
$$

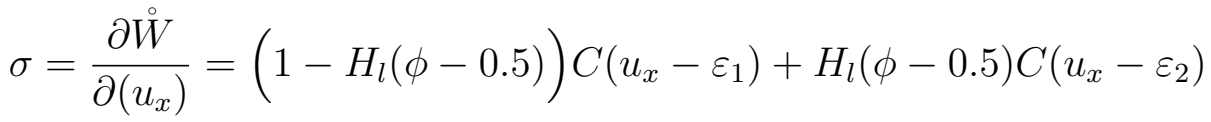

$$
\begin{aligned}
& f=\delta_{l}(\phi-0.5)\left(\frac{1}{2} C\left(u_{x}-\varepsilon_{1}\right)^{2}-\frac{1}{2} C\left(u_{x}-\varepsilon_{2}\right)^{2}\right)+\epsilon \phi_{x x} \\
& \rho \ddot{u}=\sigma_{x} \\
& \dot{\phi}=\left|\phi_{x}\right| v_{n}^{\phi}
\end{aligned}
$$

The stored energy density near each well is quadratic with wells at $\varepsilon_{1}=0$ and $\varepsilon_{2}=1$.

We examine three different kinetic laws:

$$
\hat{v}_{n}^{\phi}= \begin{cases}\operatorname{sign}(f) \kappa|f| & \text { linear kinetics } \\ \operatorname{sign}(f) \kappa|f|^{2} & \text { quadratic kinetics } \\ 0 \text { if }|f|<f_{0} \text { else } \operatorname{sign}(f) \kappa \cdot\left(|f|-f_{0}\right) & \text { stick-slip kinetics }\end{cases}
$$

and test if the direct dynamic simulations show a similar relation between interface velocity and classical driving force.

The configuration is a 1D bar with a phase interface at the center of the bar. The bar is fixed at the left end and a constant load $P$ is applied at the right end. This causes an elastic wave to head towards the left from the right end. When the elastic wave hits the phase interface, it causes the interface to begin moving. Repeated calculations over a range of applied loads causes interfaces to propagate at a range of velocities.

Fig. 6 shows the evolution of the interface after the elastic wave hits it in the case of linear kinetics. It can be seen that the solution quickly reaches a steady-state evolution. The quadratic kinetics and the stick-slip kinetics above the sticking threshold display qualitatively similar evolution.

We note in Fig. 7 (left) the attractive feature of the model that the driving force field is constant in the vicinity of the interface. Consequently, $v_{n}^{\phi}$ is constant in that region, enabling the transport of the interface density without distortion of the interface shape. This also enables clear physical interpretations of the notion of driving force and interface velocity. To find the induced kinetics, we compute the classical driving force and plot it against the interface velocity. Fig. 7 (right) shows the induced kinetics for the kinetic response functions in (4.6). 

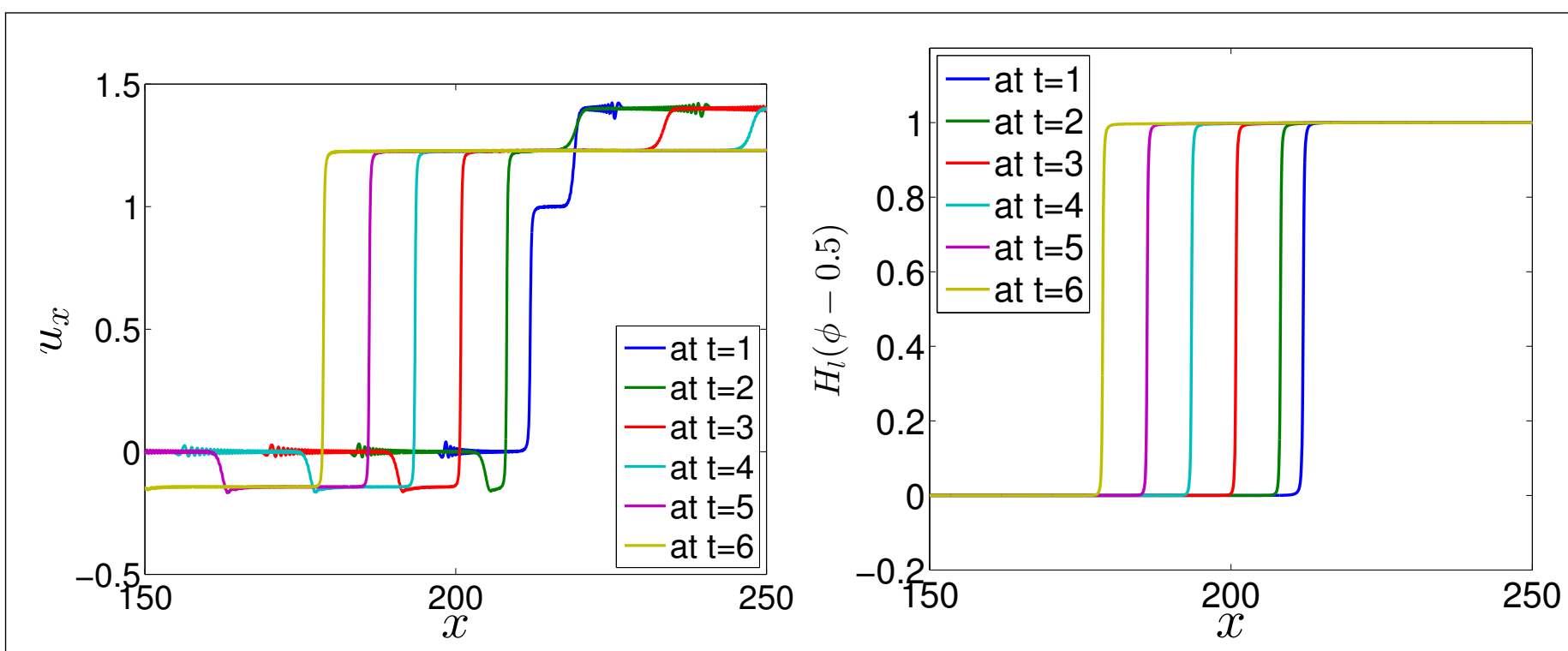

Figure 6: $u_{x}$ (left) and $H_{l}(\phi(x)-0.5)$ at different times after the elastic wave hits the phase interface, showing the steady state evolution of the interface.
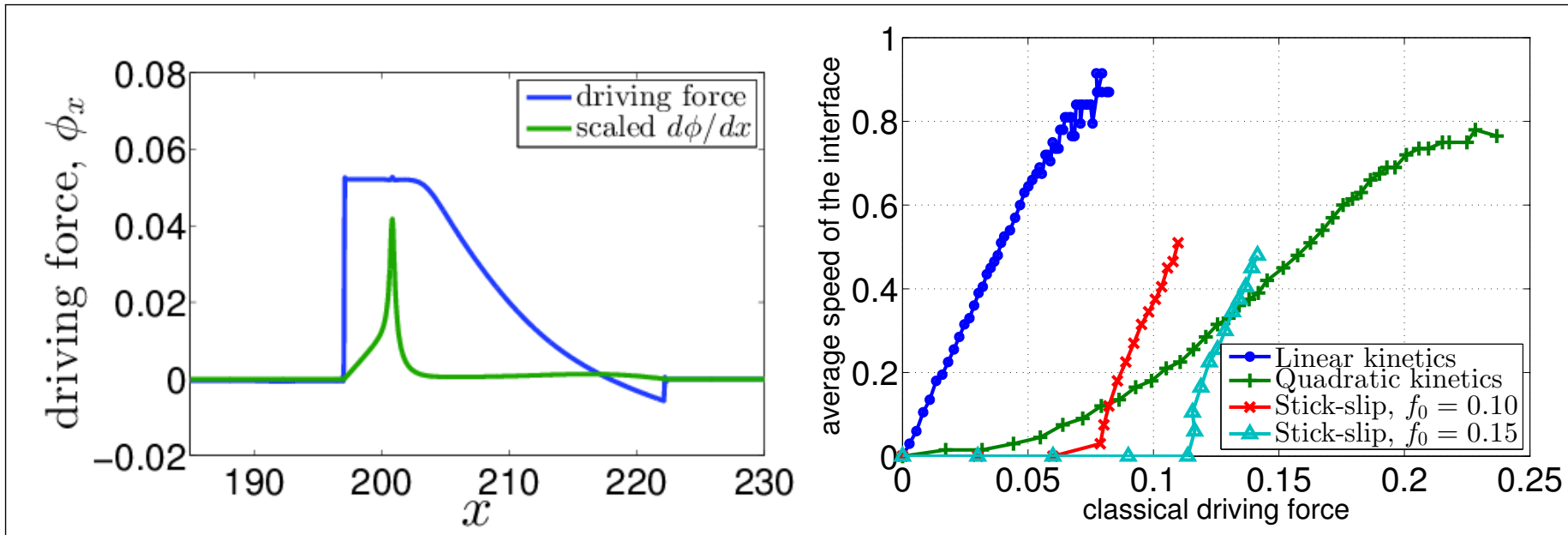

Figure 7: Left: Driving force in the vicinity of the interface, showing that it constant. The interface is moving towards the left. Right: Interface velocity vs. classical driving force for different kinetic laws.

The induced kinetic relation follows quite well the kinetic response functions in (4.6) but the agreement gets worse as $M \rightarrow 1$. This is to be expected since balance of linear momentum does not permit supersonic interfaces irrespective of the driving force.

The stick-slip kinetic response permits evolution only if driving force exceeds a threshold value, and we see the same induced behavior in terms of classical driving force. The precise threshold value is different, but the ratio of the threshold value is preserved for the two stick-slip kinetic laws that were tested in Fig. 7 (right).

Fig. 8 compares the kinetics derived from traveling wave solutions and direct dynamic simulations. For 
the same values of all parameters, the curves lie on top of each other, except near the sonic velocity where a steady traveling takes an extremely long time to develop. Therefore, it is more useful to compare with slightly different kinetics, and we observe that there is qualitative similarity between the traveling wave solutions and the dynamic simulations.

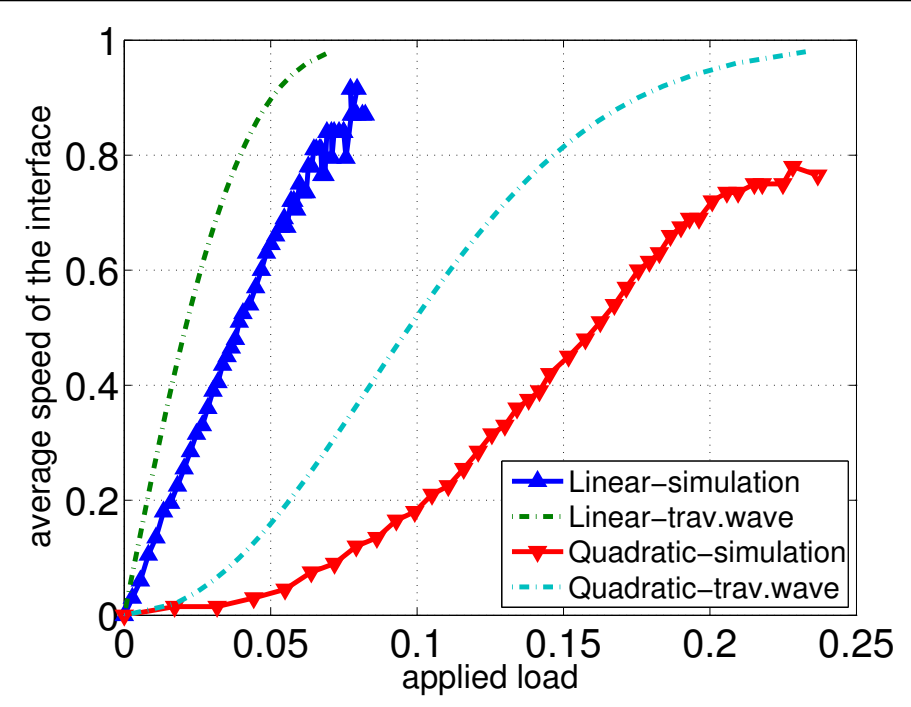

Figure 8: Comparison of linear and quadratic kinetics from dynamic simulations and traveling wave solutions. The chosen kinetic relations have different coefficients for the dynamics and traveling wave cases.

\section{Effect of the Small Parameter $l$}

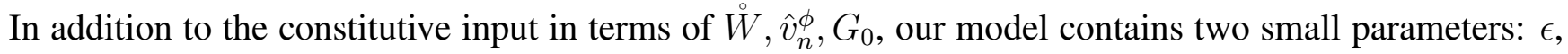
the coefficient of $|\nabla \phi|^{2}$, and $l$, the parameter in the regularized Heaviside-like function $H_{l}$ (see Fig. 1). There is a good physical understanding of $\epsilon$ as being related to the thickness of phase interfaces. We note that $l$ provides a measure of the size (in strain space) of the unstable region between the stable phases, but the precise role in determining the induced kinetic relations is unclear. We examine this role by computing the induced kinetic relations for various values of $l$. We use both traveling waves with linear kinetics and dynamic calculations with a stick-slip kinetic response. We use $\stackrel{\circ}{W}$ as in Section 4.

Fig. 9 shows that the kinetics is quite sensitive to $l$. Ideally, we would like to see if there is convergence in any sense as $l \rightarrow 0$, but the energy is extremely steep as $l$ becomes smaller and does not permit numerical simulations with confidence. From the calculations that we could confidently carry out, there appears to be no such convergence. However, while the kinetics is sensitive to $l$, the essential effect seems to be as a pre-multiplying coefficient that does not affect the shape of the kinetic response function. Therefore, a simple strategy to deal with this is to fix a given value of $l$ that allows easy numerical simulations, and then calibrate the pre-multiplier in the kinetic response function to the desired value based on this fixed value of $l$. In other words, treat $l$ as a fixed material parameter. In some ways, this is reminiscent of the behavior of standard regularized models in which the observed kinetics is very sensitive to the regularization parameters; the key difference is that the precise role of the regularization in setting the kinetics is typically opaque. 
Additionally, an interesting observation from the dynamic calculations is that the relation between interface velocity and applied end load is fairly insensitive to $l$.

The linear kinetic relation in Fig. 9 also shows an interesting feature in relation to the competition between the linear kinetic response and the inability of the interface to go beyond $M=1$. As $l \rightarrow 0$, we note that the kinetic relation remains linear for higher $M$; this issue is further discussed in Section 6.
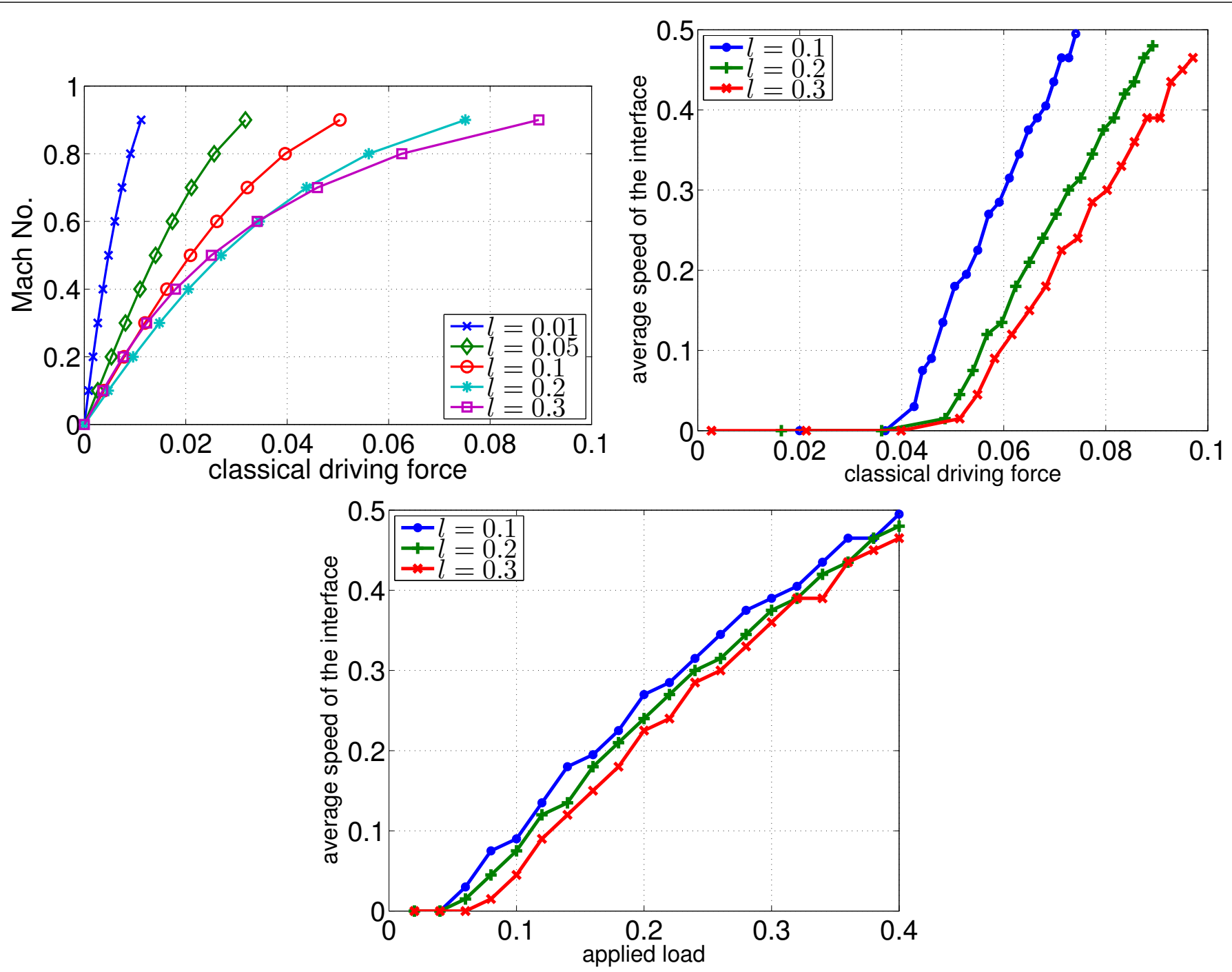

Figure 9: Left: Interface velocity vs. classical driving force with linear kinetics computed using traveling waves for different values of $l$. Right: Interface velocity vs. classical driving force with stick-slip kinetics computed using dynamic calculations for different values of $l$. Below: Interface velocity vs. applied end load for the same dynamic calculations.

\section{Competition Between Inertia and Dissipation}

Phase interfaces in continuum mechanics provide an interesting demonstration of the competition between inertia and dissipation. Consider elastic shocks or their analog in gasdynamics: the behavior of 
these interfaces is almost completely constrained by momentum balance. Thermodynamics - in the sense of positive dissipation - typically serves only to select one of two possible solutions permitted by momentum balance. Typical phase interfaces are quite different from elastic shocks. Momentum balance provides only a weak constraint on the solutions, and there is a massive non-uniqueness that is left open. Non-equilibrium thermodynamics - in the form of a kinetic relation - selects the unique solution.

Regularized models of elastic shocks and phase interfaces also display this character. The addition of viscosity and gradient terms serves to regularize elastic shocks, but does not significantly change the kinetics if these regularizing mechanisms are sufficiently small. On the other hand, viscosity and gradient terms completely determine the kinetics of phase interfaces regardless of how small they may be. If one thinks of viscosity and these higher-order terms as related to dissipation, we see again the contrast between elastic shocks and phase interfaces.

In our model, this interplay may be observed in 2 ways. First, we recover the classical continuum driving force only in the quasistatic limit without inertia ${ }^{4}$. Second, when we compare the prescribed kinetic response with the observed relation between classical driving force and interface velocity, we find that the disagreement becomes larger as we approach the sonic velocity. Therefore, it is reasonable to consider the Mach number $M$ as a measure of the relative dominance of inertia vs. dissipation; $M=0$ corresponds to dissipation-dominance, and $M=1$ corresponds to inertia-dominance ${ }^{5}$.

This brings us to an interesting example studied by [AK91b, Ros95]. Consider a 1D problem with the material model shown in Fig. 10. Let the elastic modulus of phase 1 be less than the modulus of phase 3, but let them have the same mass density. Therefore, the sonic speeds in these phases satisfy $c_{1}<c_{3}$. For an interface moving at velocity $v$, define $M_{1}:=v / c_{1}$ and $M_{3}:=v / c_{3}$. Note that $M_{3}<M_{1}$ for all $v$.

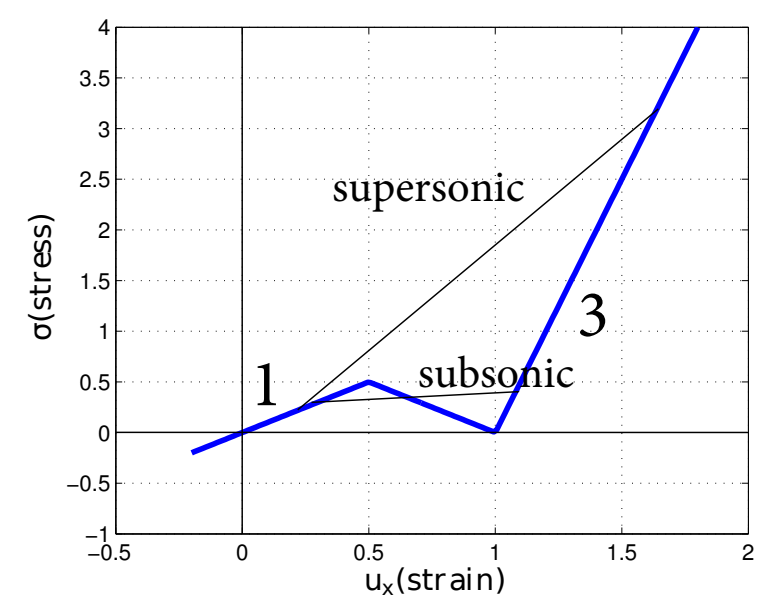

Figure 10: The stress-strain curve showing phases 1 and 3. The chords link the states on either side of the phase interface. The upper chord is a supersonic interface, and the lower chord is subsonic. The slope of a chord gives the interface velocity, and the slope of a stress-strain branch gives the sonic speed for that branch.

[AK91b, Ros95] consider, among various topics, a phase interface that bridges the phases 1 and 3. To

\footnotetext{
${ }^{4}$ Further assumptions are necessary, but not relevant to this discussion.

${ }^{5}$ We note also from Fig. 9 that smaller values of $l$ correspond to increased dissipation-dominance.
} 
briefly summarize their findings regarding this problem, they find that momentum balance permits this phase interface to have $M_{1}>1$ if it is propagating into phase 3 . Further, when $M_{1}<1$, then the interface requires a kinetic relation for unique evolution, but when $M_{1}>1$ the interface evolution is fully determined by momentum balance. Note that $M_{1}<1$ is required when the interface propagates into phase 1 , and $M_{3}<1$ always.

Therefore, an important challenge for the model that we have proposed is whether it can naturally capture this transition from dissipation-dominated evolution to inertia-dominated evolution. In other words, suppose we perform a dynamic calculation in which the velocity of the interface starts off subsonic, but at some point becomes supersonic. Will the model "automatically" know that the interface should not be governed by the kinetic response once it transitions to supersonic?

We examine this question using a combination of traveling wave analysis and dynamic calculations. We work with the following model:

$$
\begin{aligned}
\stackrel{\circ}{W}\left(u_{x}, \phi\right) & =\left(1-H_{l}(\phi-0.5)\right) \frac{1}{2} C_{1} u_{x}^{2}+H_{l}(\phi-0.5) \frac{1}{2} C_{3}\left(u_{x}-\varepsilon_{3}\right)^{2} \\
\sigma & =\frac{\partial \stackrel{\circ}{\partial}\left(u_{x}\right)}{2}=\left(1-H_{l}(\phi-0.5)\right) C_{1} u_{x}+H_{l}(\phi-0.5) C_{3}\left(u_{x}-\varepsilon_{3}\right)
\end{aligned}
$$

where the stress-free strains are 0 and $\epsilon_{3}>0$. The elastic moduli are $C_{1}, C_{3}$ with $C_{1}<C_{3}$.

We use the traveling wave ansatz $u(x, t)=U(x-V t), \phi(x, t)=\Phi(x-V t), \sigma(x, t)=\Sigma(x-V t)$ and similarly for other fields, with $V>0$. Integrating $\rho \ddot{u}=\frac{d \sigma}{d x} \Rightarrow \rho V^{2} U^{\prime \prime}=\Sigma^{\prime}$ once, we get:

$$
\rho V^{2} U^{\prime}=\left(1-H_{l}(\Phi-0.5)\right) C_{1} U^{\prime}+H_{l}(\Phi-0.5) C_{3} \cdot\left(U^{\prime}-\varepsilon_{3}\right)+\text { const. }
$$

Using $M_{1}=\frac{V}{\left(C_{1} / \rho\right)^{\frac{1}{2}}}$ and $M_{3}=\frac{V}{\left(C_{3} / \rho\right)^{\frac{1}{2}}}$ and collecting the terms multiplying $U^{\prime}$, we get:

$$
U^{\prime}=\frac{C-\epsilon_{3} M_{1}^{2} H_{l}(\Phi-0.5)}{M_{3}^{2}\left(M_{1}^{2}-1\right)-\left(M_{1}^{2}-M_{3}^{2}\right) H_{l}(\Phi-0.5)}
$$

We can get 3 useful results from (6.4):

1. Consider that $\left.\Phi\right|_{-\infty}=0,\left.\Phi\right|_{+\infty}=1$, and define $\left.\epsilon^{-} \equiv U^{\prime}\right|_{-\infty},\left.\epsilon^{+} \equiv U^{\prime}\right|_{+\infty}$. Evaluating (6.4) at $\pm \infty$ and subtracting gives $\epsilon^{+}-\epsilon^{-}=\epsilon_{3} \frac{M_{1}^{2}}{M_{3}^{2}} \frac{1}{1-M_{1}^{2}}$. The jump in $\epsilon$ is positive if $M_{1}<1$ and negative if $\left|M_{1}\right|>1$. From the fact that we have phase 1 on the left, the jump in $\epsilon$ is expected to be positive.

2. Consider precisely $M_{1}=1$. We have $U^{\prime}=\frac{C-\epsilon_{3} H_{l}(\Phi-0.5)}{-\left(1-M_{3}^{2}\right) H_{l}(\Phi-0.5)}$. Since $\left.\Phi\right|_{-\infty}=0$, we require that $C=0$ for this case to have $U^{\prime}$ bounded. Therefore, $U^{\prime}=\frac{-\epsilon_{3} H_{l}(\Phi-0.5)}{-\left(1-M_{3}^{2}\right) H_{l}(\Phi-0.5)}$, which implies that at a given spatial location, either (i) $U^{\prime}=\frac{\epsilon_{3}}{1-M_{3}^{2}}$, or (ii) $H_{l}(\Phi-0.5)=0$. These conditions imply that the strain is constant in the vicinity of an interface in $\Phi$, and the strain can transition from one phase to another only away from an interface in $\Phi$. Our dynamic calculations, described below, show this feature that the interfaces in the $U$ field and the $\Phi$ field are at different spatial locations as $M_{1} \rightarrow 1$. It appears that the system responds to over-constraining by this mechanism of separating the evolution of $\phi$ from the evolution of $u$.

3. Recall that $M_{3}<M_{1}$, and examine the denominator in (6.4) for $M_{1}<1$ and $M_{1} \geq 1$. For all values of $M_{1}<1$, the denominator is positive and therefore $U^{\prime}$ is bounded everywhere. On the 
other hand, when $M_{1} \geq 1$, the denominator goes to 0 when $H_{l}(\Phi-0.5)=\frac{M_{3}^{2}\left(M_{1}^{2}-1\right)}{M_{1}^{2}-M_{3}^{2}}=\frac{M_{1}^{2}-1}{M_{1}^{2} / M_{3}^{2}-1}$. Using that $M_{3}<1$, we have that $0<\frac{M_{1}^{2}-1}{M_{1}^{2} / M_{3}^{2}-1}<1$. Noting that $H_{l}$ takes values between 0 and 1 , it follows that the condition $H_{l}(\Phi-0.5)=\frac{M_{1}^{2}-1}{M_{1}^{2} / M_{3}^{2}-1}$ is satisfied at some spatial location(s) for every $M_{1} \geq 1$. Therefore, our model will display unbounded strain at some point in the domain for interfaces that propagate at $M_{1}>1$.

We now examine this question through direct dynamic calculations. While the traveling wave framework has already ruled out existence of supersonic interfaces in our model, it is based on the assumption of steadily-propagating interfaces. Dynamic simulations enable us to probe the transient behavior as interfaces accelerate towards the sonic speed. Essentially, we expect that the dynamics will not tend to a steady traveling wave state because such a state has been shown above to not exist.

We consider a material model with $C_{3} / C_{1}=2.25 \Rightarrow M_{3}=M_{1} / 1.5$. Fig. 11 (top) shows the initial state with a stationary interface and a compressive shock approaching from the right. The other plots in Fig. 11 show the evolution of $\phi$ and $u_{x}$. We find that (1) the interface in $\phi$ moves extremely rapidly, and is in fact significantly above sonic with respect to all wave speeds in the problem!, but (2) the interface in $u_{x}$ moves completely independently of the $\phi$-interface, and is subsonic with respect to both phases. The $\phi$-interface carries a small elastic wave with it, but this can be considered as the response to a supersonic moving external load rather than as a supersonic wave.

In conclusion, it appears that our model does not handle the transition of phase interfaces from regimes where a kinetic relation is required, to regimes where a kinetic relation would overconstrain the evolution. Our model simply does not allow supersonic transitions of the type studied in [AK91b, Ros95]. However, it is encouraging that the model does not produce spurious seemingly-realistic solutions, but provides some warning. That is, if we observe that the interfaces in $\phi$ and $u$ do not have the same spatial location, or if there are no steady traveling wave-like solutions, it is likely that the evolution is over-constrained and has transitioned from dissipation-dominated to inertia-dominated.

We show in Appendix B that the standard phase-field models suffers from this same deficiency.

Finally, we note another example of the tension between inertia and dissipation: in [PB03], they find that certain phase interfaces in continuum string models also do not require an additional kinetic relation for unique evolution.

\section{Discussion}

We have presented the formulation and characterization of a phase-field model that has non-singular interfaces yet allows for transparent prescription of kinetics and nucleation of interfaces. The key elements are a re-parametrization of the energy and an evolution law that enables us to separate nucleation from kinetics. In standard phase-field approaches, these are mixed together in an extremely opaque manner. For instance, a uniform phase can nucleate a new phase through the kinetic equation, and there is no separate nucleation equation. This mixing between kinetics and nucleation makes calibration extremely challenging. In addition, standard phase-field models do not have a direct connection between evolution of $\phi$ and the kinetics of interfaces, and nucleation is completely opaque. In our formulation, the calibration of nucleation and kinetics is simple and transparent. 

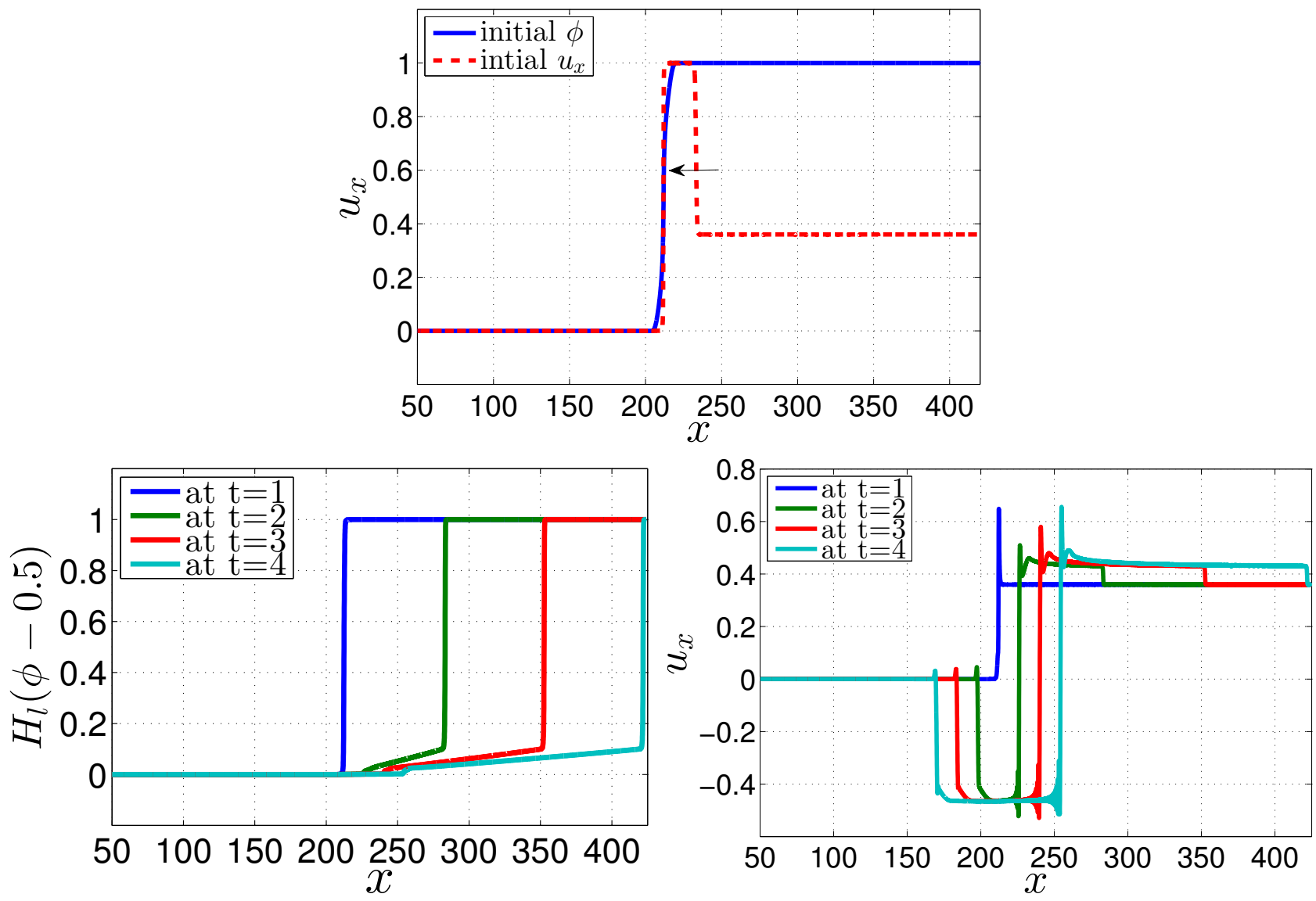

Figure 11: Top: A stationary interface as a compressive elastic wave approaches from the right. Left: The evolution of $H_{l}(\phi-0.5)$ shows the phase interface in $\phi$. Right: The evolution of $u_{x}$ shows the phase interface in $u_{x}$. The key point is that the evolution of phase interfaces in $\phi$ and $u_{x}$ are decoupled.

We find two positive indications regarding our model: (i) a formal limit of the kinetic driving force recovers the classical continuum sharp-interface driving force of Abeyaratne and Knowles; (ii) examining interfaces as traveling waves in our model, we find that the driving force is constant in space, unlike the variational derivative of standard phase-field models. The latter result implies that kinetic relations - i.e., a relation between velocity and driving force - is a well-defined notion in our model, and provides confidence in both the re-parametrized energy and the evolution statement. In addition, our re-parametrization of the energy modifies barriers in a drastic way, but preserves the structure of the energy away from the barriers. However, we recall that the driving force in the classical setting [AK06] depends only on the end-states on either side of the interface and not on the details of the barrier. The information about the barrier is retained in our model through the kinetics and nucleation that we prescribe.

In the companion paper, we present a number of examples characterizing our model in two dimensions.

Our approach could be criticized as introducing extra equations and parameters that are not used in traditional phase-field models. Is this a disadvantage of our approach, since it becomes more complex, with more fitting parameters required from experiment, MD, etc.? In other words, have we sacrificed model simplicity for model flexibility? For instance, standard phase-field models with only a handful 
of parameters and energy minimization-driven kinetics have been proven remarkably effective for many applications in materials science. However, it is our opinion that while standard phase-field models are certainly very simple, that is because the many significant assumptions related to kinetics and nucleation that are present are simply not made explicit. Our model could be made just as simple, for instance, by assuming that we have linear kinetics and that nucleation occurs at some arbitrarily-chosen value of the driving force. This would provide a model that is just as simple and easy to formulate as a standard phase-field model. But even in the setting that we wish to use only a handful of parameters, our model makes transparent the assumptions that we are making on kinetics and nucleation.

An important open problem for our formulation is the inability to handle supersonic phase interfaces that do not require kinetics to obtain a unique evolution; in fact, prescription of kinetics overconstrains the evolution in the classical setting. We find that our model cannot describe such supersonic interfaces in a traveling-wave setting corresponding to steady propagation, and dynamic calculations show strange behavior in these settings. We further find that standard phase-field models also have this deficiency. Regularized models that are based on adding viscosity and strain-gradients to the stress-response can correctly describe this interface [Ros95]. But strain-gradient models have an important disadvantage compared to phase-field models. Strain-gradient models do not use a phase-field at all but work exclusively with the displacement field. The strain-gradients then impose severe smoothness requirements on the displacement field, making it challenging for finite elements in higher dimensions. In contrast, phasefield models - both the existing approaches as well as our formulation - require only the usual levels of smoothness because the gradients are on $\phi$ and only of second-order, and finite element implementations are commonplace, e.g. [XB08, SL07, YD12].

Ongoing extensions of this work are as follows:

1. The extension to 3 or more phases is straightforward following the ideas presented here. This is an important capability for realistic problems, and is a focus of our ongoing work.

2. Our model extends the domain of stable phases, and allows them to exist in regions of strain space that should correspond to unstable phases. This is not an ideal situation, and our ongoing work aims to remedy this by appropriately using nucleation criteria.

3. Phase-field models for fracture are gaining in popularity. Our ongoing work aims to extend the work presented here to that setting to enable dynamic fracture calculations. In addition, we find that traveling wave analyses of the type presented in Appendix B are providing much insight into the behavior of supersonic cracks in the existing phase-field models of fracture, e.g. [BVS ${ }^{+} 12$ ].

4. We have used finite elements for the elasticity and finite differences for the evolution of $\phi$. However, the evolution of $\phi$ comes from a conservation principle, which can provide important advantages for robust numerical implementations such as a natural weak form [Tra09]. The development of such a scheme may be a useful future extension.

\section{Acknowledgments}

We thank ARO (W911NF-10-1-0140) for financial support. Vaibhav Agrawal also thanks the Carnegie Mellon University College of Engineering for financial support through the Bertucci Graduate Fellowship. Kaushik Dayal also thanks the Carnegie Mellon University College of Engineering for financial 
support through the Early Career Fellowship. This research was also supported in part by the National Science Foundation through TeraGrid resources provided by Pittsburgh Supercomputing Center. We thank Phoebus Rosakis for many useful discussions, encouragement, and pointers to the literature. We also thank the anonymous reviewers for many useful suggestions, including the suggestion to split the original single manuscript into two papers.

\section{A Connection to Noether's Theorem}

A seminal observation by Noether was that conservation principles in physics often have their roots in continuous symmetries. For instance, conservation principles for linear momentum, angular momentum, and energy can be shown to arise from the facts that the energy of an isolated body is independent of translations in space, rotations in space, and translations in time respectively. A concrete and extremely useful manifestation of Noether's principle is in [KS72], where they show that the essential path-independent properties of the $J$-integral of fracture mechanics are related to the application of Noether's theorem. The idea can be roughly summarized as the procedure: (i) find the Lagrangian or action corresponding to the equation, and then (ii) take the variation with respect to the translations mentioned above.

We briefly examine here if a similar procedure provides any insight into the the conservation principle for interfaces. For simplicity, we consider the one-dimensional setting with the interface velocity field being a constant that we set to 1 , i.e. our evolution equation is simply $\phi_{t}=\phi_{x}$. Write the action $A=\iint \frac{1}{2}\left(\phi_{t}^{2} \phi_{x}-\phi_{x}^{2} \phi_{t}\right) d x d t$. We have constructed $A$ by trial-and-error, using [Whi65] as a starting point. Subscripts $x$ and $t$ refer to space and time derivatives respectively. We take the variation of $A$ by using the perturbation $\phi \rightarrow \phi+\epsilon \eta$, and evaluating $\left.\frac{d A}{d \epsilon}\right|_{\epsilon=0}=0$ provides:

$$
\phi_{t} \phi_{x t}-\phi_{x x} \phi_{t}-\phi_{x} \phi_{x t}+\phi_{t t} \phi_{x}+\phi_{t} \phi_{x t}-\phi_{x} \phi_{x t}=0
$$

We have assumed that $\phi$ is sufficiently smooth for all derivatives to exist.

The Euler-Lagrange equation is a very nonlinear PDE and possibly has non-unique solutions. However, we observe that $\phi_{t}=\phi_{x}$ is a solution, and also $\phi_{t}=\phi_{x} \Rightarrow \phi_{t t}=\phi_{x x}=\phi_{x t}$. Further, we observe that $\phi_{t t}=\phi_{x x}$ by itself, without assuming $\phi_{t}=\phi_{x}$, does not appear to be a solution. Therefore, this action is promising in that it provides the first-order wave equation but not the second-order equation. The Hamiltonian is computed from the Legendre transform $\int\left(\phi_{t} \frac{\partial L}{\partial \phi_{t}}-L\right) d x$ to be $\frac{1}{2} \phi_{t}^{2} \phi_{x}$.

We examine then the effect of enforcing invariance under translations of the form $t \rightarrow t+\epsilon$ and $x \rightarrow x+\epsilon$. Unfortunately, these do not provide any further insights. This is not too surprising, because Noether's theorem typically does not provide new evolution laws, but rather can provide conserved quantities that are already implied by the original evolution laws; e.g., given the force-acceleration equation for an isolated system of particles, Noether's principle can show that the total momentum is conserved. 


\section{B Non-existence of Supersonic Interfaces in Standard Phase-field Models}

We consider a 1D phase-field model with energy as follows:

$$
\int_{\Omega} W(\phi)+\frac{1}{2} C(\phi)\left(\epsilon-\epsilon_{0}(\phi)\right)^{2}+\frac{1}{2} \epsilon\left(\frac{\partial \phi}{\partial x}\right)^{2}
$$

where $W$ is a nonconvex energy density with multiple wells. Using the standard gradient-flow assumption, the evolution equations are:

$$
\rho \ddot{u}=\frac{\partial}{\partial x}\left[C(\phi)\left(\epsilon-\epsilon_{0}(\phi)\right)\right], \quad \dot{\phi}=\frac{d W}{d \phi}+\epsilon \frac{\partial^{2} \phi}{\partial x^{2}}
$$

The context of the calculation here is based on the discussion in Section 6. Analysis of classical continuum models [AK90] and strain-gradient models [Ros95] show the possibility of interfaces that are supersonic with respect to the softer phase. But our model does not admit such interfaces, and requires that interfaces are subsonic with respect to both phases. We examine this question in the context of the standard phase-field model (B.1), (B.2). We note that setting the elastic modulus $C(\phi)>0$ to be an explicit function of the phase parameter allows the moduli - and hence the sonic velocities - of the phases to be different.

We now analyze just the momentum balance equation from (B.2). Assuming a traveling wave framework, we write $u(x, t)=U(x-V t), \phi(x, t)=\Phi(x-V t)$. This gives us $\rho V^{2} U^{\prime \prime}=\left[C(\Phi)\left(U^{\prime}-\epsilon_{0}(\Phi)\right)\right]^{\prime}$. Integrating once, we get $\rho V^{2} U^{\prime}=C(\Phi)\left(U^{\prime}-\epsilon_{0}(\phi)\right)+D$. We rearrange to write:

$$
U^{\prime}=\frac{D-\epsilon_{0}(\Phi)}{1-M^{2}(\Phi)}
$$

where $M(\Phi):=\frac{V}{(C(\Phi) / \rho)^{\frac{1}{2}}}$ is the local Mach number.

Let $C(\phi)$ be a continuous function of its argument $\phi^{6}$. It follows that $M(\Phi)$ is a continuous function of $\Phi$. Now, consider a phase interface; such an interface involves a transition from $\Phi=0$ to $\Phi=1$, with $\Phi$ being continuous in its argument. Therefore, if $M(\Phi)>1$ at any point in space corresponding to the softer phase, and $M(\Phi)<1$ at any point in space corresponding to the stiffer phase, then $M=1$ at some point in the domain. Further, $U^{\prime}$ is unbounded at the point where $M=1$. Hence, phase-field models cannot support steadily-propagating phase interfaces that are supersonic with respect to the softer phase.

\section{References}

[AA12] Amir Abdollahi and Irene Arias, Phase-field modeling of crack propagation in piezoelectric and ferroelectric materials with different electromechanical crack conditions, Journal of the Mechanics and Physics of Solids 60 (2012), no. 12, 2100-2126.

\footnotetext{
${ }^{6}$ Assuming that $C(\phi)$ is not continuous would require us to explicitly track the discontinuity in numerical calculations, thereby destroying the essential advantage of phase-field approaches.
} 
[Ach01] Amit Acharya, A model of crystal plasticity based on the theory of continuously distributed dislocations, Journal of the Mechanics and Physics of Solids 49 (2001), no. 4, 761-784.

[AD14] Amit Acharya and Kaushik Dayal, Continuum mechanics of line defects in liquid crystals and liquid crystal elastomers, Quarterly of Applied Mathematics 72 (2014), no. 1, 33-64.

[AK90] Rohan Abeyaratne and James K Knowles, On the driving traction acting on a surface of strain discontinuity in a continuum, Journal of the Mechanics and Physics of Solids 38 (1990), no. 3, 345-360.

[AK91a]__ Implications of viscosity and strain-gradient effects for the kinetics of propagating phase boundaries in solids, SIAM Journal on Applied Mathematics 51 (1991), no. 5, 12051221.

[AK91b] K Kinetic relations and the propagation of phase boundaries in solids, Archive for rational mechanics and analysis 114 (1991), no. 2, 119-154.

[AK06] _ Evolution of phase transitions: a continuum theory, Cambridge University Press, 2006.

[Ant05] Stuart S Antman, Nonlinear problems of elasticity, vol. 107, Springer, 2005.

[BCR10] Nathalie Bozzolo, Lisa Chan, and Anthony D Rollett, Misorientations induced by deformation twinning in titanium, Journal of Applied Crystallography 43 (2010), no. 3, 596-602.

[BEKT12] CD Barrett, Haitham El Kadiri, and MA Tschopp, Breakdown of the schmid law in homogeneous and heterogeneous nucleation events of slip and twinning in magnesium, Journal of the Mechanics and Physics of Solids 60 (2012), no. 12, 2084-2099.

[Bha03] Kaushik Bhattacharya, Microstructure of martensite: why it forms and how it gives rise to the shape-memory effect, vol. 2, Oxford University Press, 2003.

[BT10] IJ Beyerlein and CN Tomé, A probabilistic twin nucleation model for hep polycrystalline metals, Proceedings of the Royal Society A: Mathematical, Physical and Engineering Science 466 (2010), no. 2121, 2517-2544.

[BVS ${ }^{+}$12] Michael J Borden, Clemens V Verhoosel, Michael A Scott, Thomas JR Hughes, and Chad M Landis, A phase-field description of dynamic brittle fracture, Computer Methods in Applied Mechanics and Engineering 217 (2012), 77-95.

[Che02] Long-Qing Chen, Phase-field models for microstructure evolution, Annual review of materials research 32 (2002), no. 1, 113-140.

[CSWRS09] J Cai, S Shekhar, J Wang, and M Ravi Shankar, Nanotwinned microstructures from low stacking fault energy brass by high-rate severe plastic deformation, Scripta Materialia 60 (2009), no. 8, 599-602.

[DB06] Kaushik Dayal and Kaushik Bhattacharya, Kinetics of phase transformations in the peridynamic formulation of continuum mechanics, Journal of the Mechanics and Physics of Solids 54 (2006), no. 9, 1811-1842. 
[DWWR14] NP Daphalapurkar, JW Wilkerson, TW Wright, and KT Ramesh, Kinetics of a fast moving twin boundary in nickel, Acta Materialia 68 (2014), 82-92.

[EC93] JC Escobar and RJ Clifton, On pressure-shear plate impact for studying the kinetics of stress-induced phase transformations, Materials Science and Engineering: A 170 (1993), no. $1,125-142$.

[Eri75] JL Ericksen, Equilibrium of bars, Journal of elasticity 5 (1975), no. 3, 191-201.

[FG94] Eliot Fried and Morton E Gurtin, Dynamic solid-solid transitions with phase characterized by an order parameter, Physica D: Nonlinear Phenomena 72 (1994), no. 4, 287-308.

[FM06] Cristian Făciu and Alain Molinari, On the longitudinal impact of two phase transforming bars. elastic versus a rate-type approach. part ii: The rate-type case, International journal of solids and structures 43 (2006), no. 3, 523-550.

[FS11] Eilon Faran and Doron Shilo, The kinetic relation for twin wall motion in nimnga, Journal of the Mechanics and Physics of Solids 59 (2011), no. 5, 975-987.

[GF97] German Grach and Eliot Fried, An order-parameter-based theory as a regularization of a sharp-interface theory for solid-solid phase transitions, Archive for Rational Mechanics and Analysis 138 (1997), no. 4, 355-404.

[HB14] A Hunter and IJ Beyerlein, Predictions of an alternative pathway for grain-boundary driven twinning, Applied Physics Letters 104 (2014), no. 23, 233112.

[KAF09] Yashashree Kulkarni, Robert J Asaro, and Diana Farkas, Are nanotwinned structures in fcc metals optimal for strength, ductility and grain stability?, Scripta Materialia 60 (2009), no. 7, 532-535.

[KS72] James K Knowles and Eli Sternberg, On a class of conservation laws in linearized and finite elastostatics, Archive for Rational Mechanics and Analysis 44 (1972), no. 3, 187-211.

[LLSL10] CH Lei, LJ Li, YC Shu, and JY Li, Austenite-martensite interface in shape memory alloys, Applied Physics Letters 96 (2010), no. 14, 141910.

[MH94] Jerrold E. Marsden and Thomas JR Hughes, Mathematical foundations of elasticity, Courier Dover Publications, 1994.

[NRC06] J Niemczura and K Ravi-Chandar, Dynamics of propagating phase boundaries in niti, Journal of the Mechanics and Physics of Solids 54 (2006), no. 10, 2136-2161.

[OSPM14] A Ojha, H Sehitoglu, L Patriarca, and HJ Maier, Twin nucleation in fe-based bcc alloys?modeling and experiments, Modelling and Simulation in Materials Science and Engineering 22 (2014), no. 7, 075010.

[PAD14] Hossein Pourmatin, Amit Acharya, and Kaushik Dayal, A fundamental improvement to ericksen-leslie kinematics., to appear in Quarterly of Applied Mathematics (2014).

[PB03] Prashant K Purohit and Kaushik Bhattacharya, Dynamics of strings made of phasetransforming materials, Journal of the Mechanics and Physics of Solids 51 (2003), no. 3, 393-424. 
[Ros95] Phoebus Rosakis, An equal area rule for dissipative kinetics of propagating strain discontinuities, SIAM Journal on Applied Mathematics 55 (1995), no. 1, 100-123.

[SL07] Yu Su and Chad M Landis, Continuum thermodynamics of ferroelectric domain evolution: Theory, finite element implementation, and application to domain wall pinning, Journal of the Mechanics and Physics of Solids 55 (2007), no. 2, 280-305.

[SY08] YC Shu and JH Yen, Multivariant model of martensitic microstructure in thin films, Acta Materialia 56 (2008), no. 15, 3969-3981.

[Tra09] John A Trangenstein, Numerical solution of hyperbolic partial differential equations, Cambridge University Press, 2009.

[Tru82] LM Truskinovskii, Equilibrium phase interfaces, Sov. Phys. Dokl, vol. 27, 1982, pp. 551552.

[Tru93] L Truskinovsky, Kinks versus shocks, Shock induced transitions and phase structures in general media, Springer, 1993, pp. 185-229.

[Tur97] Sergio Turteltaub, Viscosity of strain gradient effects on the kinetics of propagating phase boundaries in solids, Journal of Elasticity 46 (1997), no. 1, 53-90.

[WBT10] J Wang, IJ Beyerlein, and CN Tomé, An atomic and probabilistic perspective on twin nucleation in mg, Scripta Materialia 63 (2010), no. 7, 741-746.

[Whi65] GB Whitham, A general approach to linear and non-linear dispersive waves using a lagrangian, Journal of Fluid Mechanics 22 (1965), no. 02, 273-283.

[WTAF09] T Waitz, K Tsuchiya, T Antretter, and FD Fischer, Phase transformations of nanocrystalline martensitic materials, MRS bulletin 34 (2009), no. 11, 814-821.

[XB08] Yu Xiao and Kaushik Bhattacharya, A continuum theory of deformable, semiconducting ferroelectrics, Archive for Rational Mechanics and Analysis 189 (2008), no. 1, 59-95.

[YD10] Lun Yang and Kaushik Dayal, Formulation of phase-field energies for microstructure in complex crystal structures, Applied Physics Letters 96 (2010), no. 8, 081916.

[YD12] _ Influence of strain on space-charge distribution at ferroelectric thin-film free surfaces, Acta Materialia 60 (2012), no. 19, 6457-6463.

[ZB05] W Zhang and K Bhattacharya, A computational model of ferroelectric domains. part $i$ : model formulation and domain switching, Acta materialia 53 (2005), no. 1, 185-198. 